\title{
Image Based Leaf Area Measurement Method Using Artificial Neural Network
}

\author{
Joko Siswantoro \\ Department of Informatics Engineering Universitas Surabaya \\ Surabaya, Indonesia \\ joko_siswantoro@staff.ubaya.ac.id
}

\author{
Ida Bagus Made Artadana \\ Department of Biology Universitas Surabaya \\ Surabaya, Indonesia \\ arta@staff.ubaya.ac.id
}

\begin{abstract}
Leaf area is an important parameter in plant monitoring. An automatic method for measuring leaf area is needed to obtain accurate result. In this paper, a method for automatic leaf area measurement from image is proposed. The method captured the image of leaf without object reference. Four features were extracted from the image and used as input to artificial neural network for estimating leaf area. The experiment results show that the proposed method can measure leaf area with mean absolute relative error less than $1 \%$ and less computational time.
\end{abstract}

Keywords-leaf area measurement, automatic, artificial neural network, image processing

\section{INTRODUCTION}

Leaf area plays an important role in plant monitoring, including monitoring plant growth, plant competition, and plant yield [1]. Moreover, leaf area can be used to measure the performance of mechanism occurred in a plant [2]. Traditionally, leaf area measurement can be performed manually using grid counting method [3-5] or paper weighing method [1]. These methods are simple in practice but time consuming. Furthermore, the measurement accuracy for these methods depends on the expertise of the operator.

To overcome the problems occurred in traditional leaf area measurement method, several alternative methods for leaf area measurement have been proposed. Córcoles, et al. [6] and Amiri and Shabani [7] have proposed semi-automatic methods for measuring leaf area. They manually measured the length and width of leaf and inputted the results to a computer program for estimating leaf area using general linear regression model (GLM) [6] and adaptive neuralbased fuzzy inference system (ANFIS) [7]. The methods have been validated using several types of leaves and produced high coefficient of determination. However, the accuracy of the methods is very dependent on the expertise of the operator in measuring length and width manually.

Gong, et al. [2], Lü, et al. [3], Patil and Bodhe [4], and Radzali, et al. [5] have been proposed automatic methods to measure leaf area by utilizing image processing technique. To measure leaf area, the proposed methods captured the image of measured leaf and an object reference with known area together using a camera. The image was then processed to obtain a binary image. Leaf area was calculated by comparing the number of leaf pixels and the number of object reference pixels in the binary image and then multiplying the result with actual object reference area. The proposed methods have been validated and produced low error. However, the using of object reference could lead a problem during segmentation process and finally could decrease measurement accuracy. In addition, the method proposed by Gong, et al. [2] was not fully automatic; the user intervention was still needed during segmentation process.

Therefore, an automatic leaf area measurement method which does not need object reference and user intervention need to be developed. A synergy between image processing technique and artificial neural network (ANN) can be considered for solving the problems. ANN is a nonlinear model that imitates human nervous system and has simple computation [8]. ANN has been applied to solve classification and prediction problems in several areas, such as natural produce recognition using computer vision [9], egg volume measurement using computer vision [10], biodiesel production optimization [11], and gas concentration estimation for electronic nose [12].

This paper proposes an image based leaf area measurement method using ANN. The proposed method uses length, width, area, and perimeter of leaf extracted from the image of measured leaf and used the features as input to ANN for predicting leaf area. Therefore the proposed method can be used to measure leaf area without object reference and user intervention. The rest of the paper is arranged as follow. Section 2 explains the material and the detail of all steps used in the proposed method. Section 3 describes the experimental setup for validating the proposed method. Section 4 presents experimental results and discussion. Finally, conclusion is drawn in Section 5.

\section{MATERIALS AND METHOD}

\section{A. Materials}

Hardware used in the proposed method consisted of a camera, two flat acrylic boards, an USB cable, and a computer, as shown in Fig. 1. A Logitech ${ }^{\circledR}$ HD Webcam $\mathrm{c} 270 \mathrm{~h}$ was used to capture the image of measured leaf. The camera was located on the above of measured leaf at a distance of $50 \mathrm{~cm}$ with an orientation such that the image plane is parallel to leaf surface and connected to the computer using the USB cable.

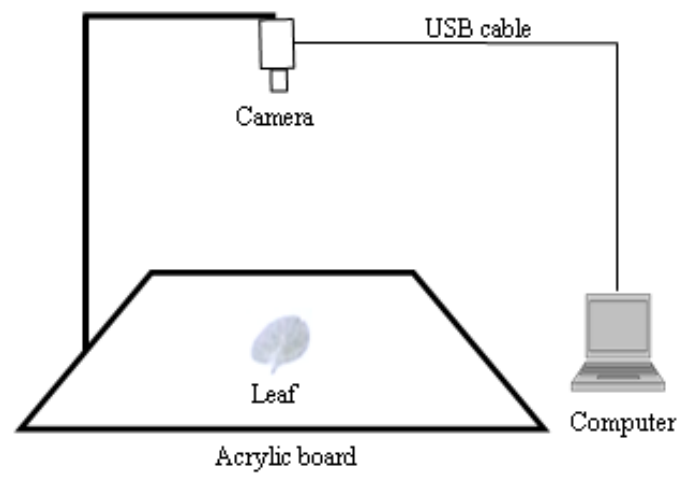


Fig. 1. Hardware used in the proposed leaf area measurement method.

To flatten the measured leaf, the leaf was placed between two flat acrylic boards. The lower board was white painted and used as background during image acquisition. The proposed system employed a $2.20 \mathrm{GHz}$ Intel Core 2 portable computer with RAM 4GB and Windows 7 operating system to control the camera, process the acquired image, and measure leaf area.

To validate the proposed method, 30 samples of leaf were chosen randomly from an area around Universitas Surabaya. The samples consisted of 10 kailan (Brassica oleracea Alboglabra Group) leaves, 10 ipecac (Cephaelis ipecacuanha) leaves, and 10 wild betel (Piper sarmentosum Roxb. Ex Hunter) leaves. The exact area of all samples were measured manually using paper weighing method [1].

\section{B. Method}

The proposed leaf area measurement method consisted of a series steps starting from image acquisition followed by preprocessing, segmentation, features extraction, and leaf area estimation. The details for every step are explained in the following subsections.

\section{1) Image Acquisition}

The measured leaf was located between two flat acrylic boards during image acquisition. The image of measured leaf was captured using the camera with a white background. The white background was chosen since in general the color of leaf is different from white. Therefore leaf pixels could be easily separated from background pixels during segmentation step. The image was captured in RGB color space and was then saved in PNG file for further process. Every leaf sample was captured twice in vertical and horizontal directions. The examples of captured image are shown in Fig. 2.

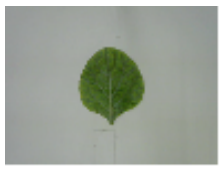

a

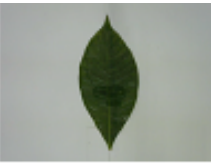

b

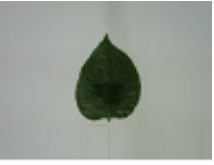

Fig. 2. The exaples of captured image: (a) kailan, (b) ipecac, (c) wild betel.

\section{2) Preprocessing}

In preprocessing step, the captured image was converted from image in RGB color space to gray scale image by calculating the weighted average of $R, G$, and $B$ values for every pixel in the captured image. To reduce noises produced by the camera, a $5 \times 5$ Gaussian filter [13] was applied to the gray scale image. Fig. 3 shows the example of preprocessing result.

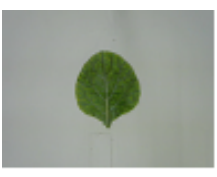

a

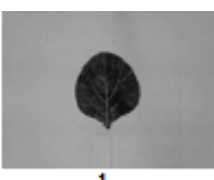

$\mathrm{b}$
Fig. 3. The example of preprocessing result: (a) captured image, (b) gray scale image.

\section{3) Segmentation}

Segmentation step was used to separate leaf pixels from background pixels in the gray scale image. The proposed method employed thresholding method [13] in this step. The threshold value $\mathrm{T}$ was automatically determined using Otsu method [14]. As can be seen in Fig. 3 (b), the intensity of leaf pixel in the gray scale image was less than the intensity of background pixel; therefore a pixel in the gray scale image with intensity less than $\mathrm{T}$ was categorized as leaf pixel with binary value 1 (white pixel), otherwise as background pixel with binary value 0 (black pixel). The result of segmentation step was a binary image, as shown in Fig. 4.

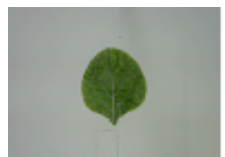

a

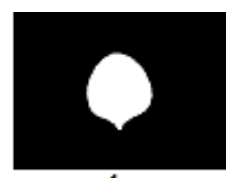

Fig. 4. The example of segmentation result: (a) captured image, (b) binary image.

\section{4) Features Extraction}

In this step, four features were extracted from leaf pixels in the binary image. The extracted features were used to estimate the area of measured leaf in the next step. The features were length, width, area, and perimeter.

- Length $(L)$ was defined as the length (in pixels) of major axis of measured leaf. The major axis was defined as line connecting between two pixels on the leaf boundary that have the longest distance in the binary image, as shown in Fig. 5 (a).

- Width $(W)$ was defined as the length (in pixels) of minor axis of measured leaf. The minor axis was defined as line connecting between two pixels on the leaf boundary that perpendicular to the major axis in the binary image, as shown in Fig. 5 (b).

- Area $(A)$ was defined as the number of leaf pixels (white pixels) in the binary image, as shown in Fig. 5 (c).

- Perimeter $(P)$ was defined as the number of pixels on the leaf boundary in the binary image, as shown in Fig. 5 (d).

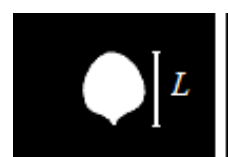

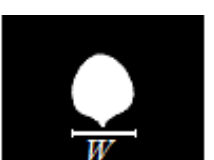

b

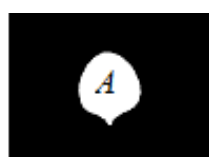

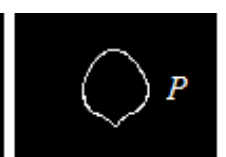

d
Fig. 5. The extracted features: (a) length, (b) width, (c) area, (d) perimeter

\section{5) Leaf Area Estimation}

To estimate the area of measured leaf, the proposed method used an artificial neural network based on the features extracted in the previous step. The architecture of ANN consisted of three layers, which are an input layer, a hidden layer, and an output layer. The output layer had one neuron that corresponds to leaf area. The number of neurons in the input layer depended on the number of features used to estimate the area of measured leaf. To obtain high leaf area estimation accuracy, a feature selection was performed by inputting all possible combination of features to ANN. Therefore, there were 15 ANNs with different input. The number of neurons in the hidden layer was heuristically determined in the range of 2 and 5 in an experiment such that the ANN produces high leaf area estimation accuracy. 
The ANN used hyperbolic tangent sigmoid, as in (1), and linear functions, as in (2), as transfer functions from the input layer to the hidden layer and from the hidden layer to the output layer, respectively. The ANN was trained using back propagation algorithm [15] with mean square error (MSE) used as performance function. All features were scaled to $[-1,1]$ before training process to avoid the domination of a feature against the others and to speed up training process [9].

$$
\begin{gathered}
f(x)=\frac{1-e^{-2 x}}{1+e^{-2 x}} \\
f(x)=x
\end{gathered}
$$

\section{EXPERIMENTAL SETUP}

To validate the proposed method, an experiment has been conducted in laboratory. In the experiment every leaf sample was captured twice in vertical and horizontal direction. Therefore, there were 20 leaf images from every type of leaf or there were 60 images data in total.

Five-fold cross validation was used to develop training and testing data from 60 leaf images [16]. All images data were randomly divided into five mutually exclusive sub data with equal size. One of sub data was used as testing data and 4 remaining sub data were used as training data. This process was repeated 5 times such that every sub data is used as testing data once.

In every training process, ANN was trained with 15 different input combinations and the different number of nodes in hidden layer. MSE between the exact area and its estimation was calculated for testing data to measure the accuracy of ANN in every training process. ANN with the lowest MSE was then used in the proposed method. In addition the training time was also considered in determining the best ANN.

Furthermore, the proposed method was used to measure the leaf area of all samples. Absolute relative error (ARE) between the exact area and the area measured using the proposed method was calculated for all samples to measure the accuracy of the proposed method. ARE was calculated using (3),

$$
A R E=\frac{\left|A_{E}-A_{P}\right|}{A_{E}} \times 100 \%
$$

where $A_{E}$ and $A_{P}$ are the exact area and the area measured using the proposed method, respectively.

\section{RESUlt AND DiscusSiON}

The proposed method was implemented in a software using Visual C++ 2010 with open source computer vision library OpenCV 231 [17]. The software was used in the experiment to measure the leaf area of all samples. The experiment results are explained in the following sub sections.

\section{A. The Architecture of ANN}

The results of feature selection and determining the number of hidden nodes in the hidden layer experiments are summarized in TABLE I. As can be seen in TABLE I. The higher accuracy with average MSE of 0.249 was achieved if ANN used $W, A$, and $P$ as input features and three nodes in the hidden layer, shown in TABLE I. The training time for such ANN architecture was 2.983 s. From TABLE I, it can also be seen that the second higher estimation accuracy with average MSE of 0.251 was obtained if ANN used L and A as input features and four nodes in the hidden layer. However, by using this architecture, there was an increase in training time of $1.504 \mathrm{~s}$.

\begin{tabular}{|c|c|c|c|c|}
\hline \multirow[b]{2}{*}{$\begin{array}{c}\text { The Number of Nodes } \\
\text { in Hidden Layer }\end{array}$} & \multicolumn{4}{|c|}{ Average MSE } \\
\hline & 2 & 3 & 4 & 5 \\
\hline \multicolumn{5}{|l|}{ Input Features } \\
\hline$L$ & 34.562 & 35.786 & 30.901 & 25.443 \\
\hline$W$ & 148.050 & 117.069 & 121.946 & 122.131 \\
\hline$A$ & 0.536 & 0.398 & 0.355 & 1.160 \\
\hline$P$ & 11.224 & 15.888 & 13.500 & 12.737 \\
\hline$L, W$ & 1.386 & 1.293 & 1.063 & 0.956 \\
\hline$L, A$ & 0.345 & 0.340 & 0.251 & 0.279 \\
\hline$L, P$ & 11.186 & 19.587 & 10.649 & 10.768 \\
\hline$W, A$ & 0.347 & 0.669 & 0.352 & 0.271 \\
\hline$W, P$ & 2.632 & 2.170 & 3.295 & 2.667 \\
\hline$A, P$ & 0.554 & 0.255 & 0.349 & 0.299 \\
\hline$L, W, A$ & 0.357 & 0.380 & 0.510 & 0.344 \\
\hline$L, W, P$ & 1.251 & 1.384 & 1.311 & 1.173 \\
\hline$L, A, P$ & 0.476 & 0.337 & 0.274 & 0.449 \\
\hline$W, A, P$ & 0.401 & 0.249 & 0.341 & 0.384 \\
\hline$L, W, A, P$ & 0.471 & 0.341 & 0.308 & 0.277 \\
\hline
\end{tabular}

TABLE I. THE RESULTS OF FEATURE SELECTION AND DETERMINING THE NUMBER OF HIDDEN NODES IN THE HIDDEN LAYER EXPERIMENTS

Based on these results, the proposed method employed two ANN architectures to estimate leaf area. The first architecture consisted of an input layer with two input nodes corespond to $L$ and $A$ features, a hidden layer with four nodes and an output layer with one node correspond to leaf area, as shown in Fig. 6. The second architecture consisted of an input layer with three input nodes corespond to $W, A$, and $P$ features, a hidden layer with three nodes and an output layer with one node correspond to leaf area, as shown in Fig. 7.

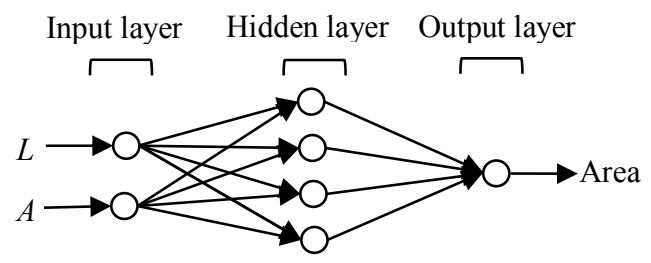

Fig. 6. The first ANN architecture used in the proposed method 


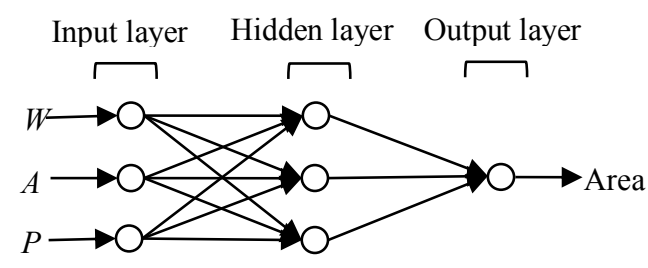

Fig. 7. The second ANN architecture used in the proposed method

\section{B. Leaf Area Measurement}

The results of leaf area measurement experiment for all samples using the proposed method with the first and the second ANN architectures are summarized in TABLE II and TABLE III, respectively. As can be seen in TABLE II and TABLE III, on average leaf area measured using the proposed method with the first and the second ANN architectures closed to exact leaf with mean ARE less than $1 \%$. This results show that the proposed method has high measurement accuracy in measuring leaf area of all samples.

TABLE II. THE MEASUREMENT RESULTS USING THE PROPOSED METHOD WITH THE FIRST ANN ARCHITECTURE

\begin{tabular}{|l|r|r|r|}
\hline \multirow{2}{*}{ Leaf Sample } & \multicolumn{2}{|c|}{ Average Area(cm $\left.{ }^{2}\right)$} & \multirow{2}{*}{$\begin{array}{c}\text { Mean } \\
\text { ARE (\%) }\end{array}$} \\
\cline { 2 - 3 } & Exact & Proposed method & \\
\hline Kailan & 29.996 & 30.060 & 0.783 \\
\hline Ipecac & 52.809 & 52.939 & 0.586 \\
\hline Wild betel & 36.896 & 36.869 & 0.939 \\
\hline All samples & 39.900 & 39.956 & 0.769 \\
\hline
\end{tabular}

TABLE III. THE MEASUREMENT RESULTS USING THE PROPOSED METHOD WITH THE SECOND ANN ARCHITECTURE

\begin{tabular}{|l|c|r|c|}
\hline \multirow{2}{*}{ Leaf sample } & \multicolumn{2}{|c|}{ Average Area $\left(\mathbf{c m}^{2}\right)$} & \multirow{2}{*}{$\begin{array}{c}\text { Mean } \\
\text { ARE (\%) }\end{array}$} \\
\cline { 2 - 3 } & Exact & Proposed method & 0.635 \\
\hline Kailan & 29.996 & 29.912 & 0.301 \\
\hline Ipecac & 52.809 & 52.802 & 0.871 \\
\hline Wild betel & 36.896 & 37.000 & 0.602 \\
\hline All samples & 39.900 & 39.905 & \\
\hline
\end{tabular}

For both ANN architectures, the lowest and highest mean ARE were achieved by the proposed method in measuring ipecac and wild betel leaves, respectively. The proposed method with the first ANN architecture produced mean ARE of $0.7796 \%$. On the other hand, with the second ANN architecture the proposed method produced lower mean ARE, which is $0.602 \%$. Based on this condition, it can be inferred that by employing the second ANN architecture the proposed method produce more accurate leaf area measurement result.

For further analysis, the linear relationship between leaf area measured using the proposed method and exact leaf area was also investigated. Fig. 8 and Fig. 9 show this linear relationship for the first and the second ANN architectures, respectively. It can be seen in Fig. 8 and Fig. 9, leaf area measured using the proposed method and exact leaf area had high linear relationship with coefficient of determination $\left(R^{2}\right)$ greater than 0.99 for both ANN architectures. It means that more that $99 \%$ variation in the exact leaf area can be explained by a linear relationship with leaf area measured using the proposed method. From computational time point of view, the proposed method only needed less than $0.1 \mathrm{~s}$ for measuring area of a leaf.

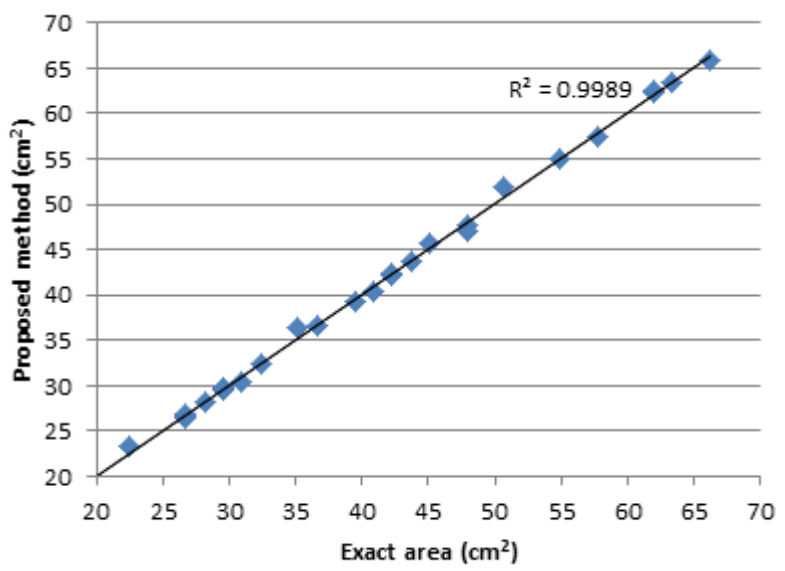

Fig. 8. The linear relationship between exact area and area measured using the proposed method with the first ANN architecture

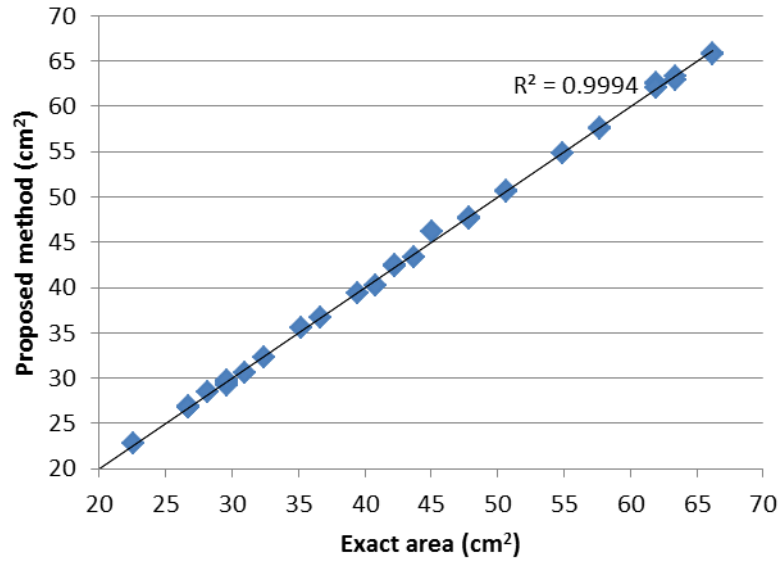

Fig. 9. The linear relationship between exact area and area measured using the proposed method with the second ANN architecture

\section{CONCLUSION}

An image based method for measuring leaf area using artificial neural network (ANN) is proposed in this paper. The method used a camera to capture the image of measured leaf from the distance of $50 \mathrm{~cm}$. The image was processed to produce a binary image. From the binary image, four features were extracted and used as input to ANN for estimating leaf area. The proposed method has been tested using three types of leaf, including kailan, ipecac, and wild betel in an experiment. The experiment results show that the proposed method has high measurement accuracy compared to exact area with mean absolute relative error less than $1 \%$. In addition, leaf area measured using the proposed method has high linear relationship with exact leaf area. Therefore the proposed method can be considered as alternative for manual measurement method in measuring leaf area. For future study, the application of the proposed method in plant growth monitoring needs to be investigated. 


\section{ACKNOWLEDGMENT}

The authors would like to thank Universitas Surabaya for providing facilities and financial support under Competitive Research Grant No. 033/Lit/LPPM-01/FT/V/2018.

\section{REFERENCES}

[1] S. K. Pandey and H. Singh, "A Simple, Cost-Effective Method for Leaf Area Estimation," Journal of Botany, vol. 2011, p. 6, 2011.

[2] A. Gong, X. Wu, Z. Qiu, and Y. He, "A handheld device for leaf area measurement," Computers and Electronics in Agriculture, vol. 98, pp. 74-80, 2013/10/01/2013.

[3] C. Lü, H. Ren, Y. Zhang, and Y. Shen, "Leaf Area Measurement Based on Image Processing," in 2010 International Conference on Measuring Technology and Mechatronics Automation, 2010, pp. 580582.

[4] S. B. Patil and S. K. Bodhe, "Betel leaf area measurement using image processing," International Journal on Computer Science and Engineering, vol. 3, pp. 2656-2660, 2011.

[5] M. H. Radzali, N. A. M. Kamal, and N. M. Diah, "Measuring Leaf Area using Otsu Segmentation Method (LAMOS)," Indian Journal of Science and Technology, vol. 9, pp. 1-6, 2016.

[6] J. I. Córcoles, A. Domínguez, M. A. Moreno, J. F. Ortega, and J. A. de Juan, "A non-destructive method for estimating onion leaf area," in Irish Journal of Agricultural and Food Research vol. 54, ed, 2015, p. 17.

[7] M. J. Amiri and A. Shabani, "Application of an Adaptive NeuralBased Fuzzy Inference System Model for Predicting Leaf Area," Communications in Soil Science and Plant Analysis, vol. 48, pp. 1669-1683, 2017.
[8] J. Siswantoro, A. S. Prabuwono, A. Abdullah, and B. Idrus, "A linear model based on Kalman filter for improving neural network classification performance," Expert Systems with Applications, vol. 49, pp. 112-122, 2016.

[9] J. Siswantoro, A. S. Prabuwono, A. Abdullah, and B. Indrus, "Hybrid Neural Network and Linear Model for Natural Produce Recognition Using Computer Vision," Journal of ICT Research and Applications, vol. 11, pp. 185-199, 2017.

[10] J. Siswantoro, M. Hilman, and M. Widiasri, "Computer vision system for egg volume prediction using backpropagation neural network," in IOP Conference Series: Materials Science and Engineering, 2017, p. 012002 .

[11] F. Kusumo, A. Silitonga, H. Masjuki, H. C. Ong, J. Siswantoro, and T. Mahlia, "Optimization of transesterification process for Ceiba pentandra oil: A comparative study between kernel-based extreme learning machine and artificial neural networks," Energy, vol. 134, pp. 24-34, 2017

[12] S. I. Sabilla, R. Sarno, and J. Siswantoro, "Estimating gas concentration using artificial neural network for electronic nose," Procedia Computer Science, vol. 124, pp. 181-188, 2017.

[13] R. C. Gonzalez and R. E. Woods, Digital Image Processing, 2nd ed.: Prentice Hall, 2002.

[14] N. Otsu, "A Threshold Selection Method from Gray-Level Histograms," Systems, Man and Cybernetics, IEEE Transactions on, vol. 9, pp. 62-66, 1979.

[15] D. E. Rumelhart, G. E. Hinton, and R. J. Williams, "Learning internal representations by error propagation," in Parallel Data Processing. vol. 1, D. Rumelhart and J. McClelland, Eds., ed Cambridge: MIT Press, 1986, pp. 318-362.

[16] E. Alpaydin, Introduction to machine learning, ke-2 ed. Cambridge, Massachusetts: MIT Press, 2010.

[17] G. Bradski, "The OpenCV Library," Dr. Dobb's Journal: Software Tools for the Professional Programmer, vol. 25, pp. 120-123, 2000 


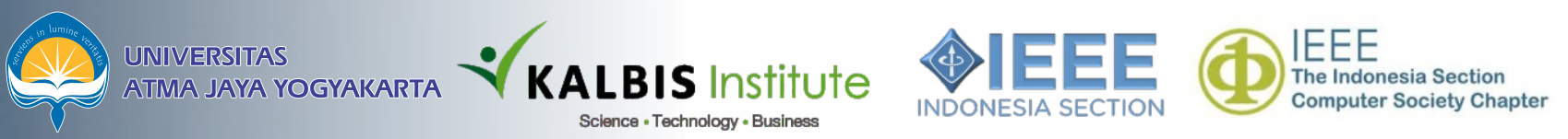

\section{INTERNATIONAL GONFERENGE OF ARIIEGLL INTELUIGENGE AND UNDOBMATION SEGHNOLOGY (10)}

]로럴 Conference Number \#45307

IEEE Catalog Number : CFP19JVE-ART ISBN

: 978-1-5386-8448-1

13 - 15 March 2019

Platinum Adisucipto Hotel $\&$ Conference Center Yogyakarta 


\section{Proceeding - 2019 International Conference of Artificial Intelligence and Information Technology, ICAIIT 2019}

COUNTRY

United States

Universities and research institutions in United States
SUBJECT AREA AND CATEGORY

\author{
Computer Science \\ Artificial \\ Intelligence \\ Computer \\ Networks and \\ Communications \\ Computer Science \\ Applications \\ Information \\ Systems \\ Signal Processing \\ Mathematics \\ Control and \\ Optimization
}

ISSN

COVERAGE

2019 
Join the conversation about this journal

\section{Prepare Your Manuscript}

We will help you write a 300-word abstract or a 30,000-word grant application. 


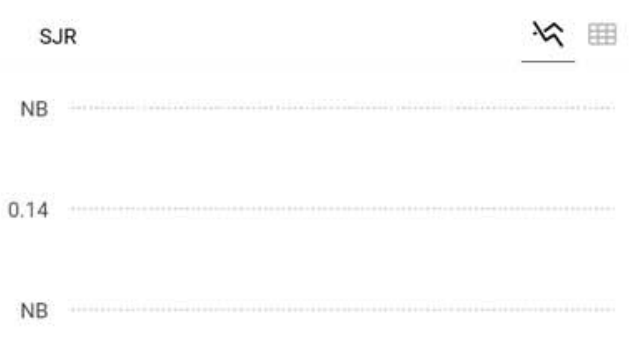

2020

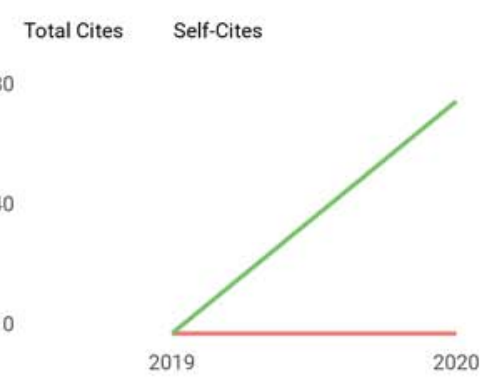

External Cites per Doc Cites per Doc 命

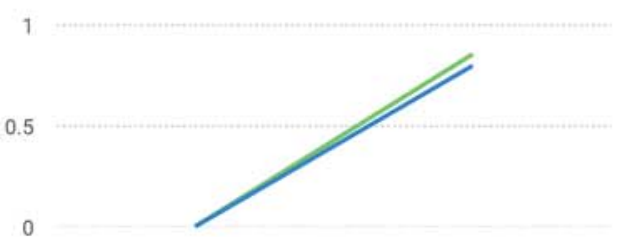

0

2019

2020

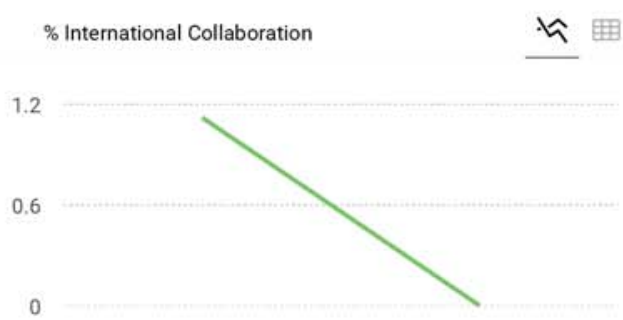

2019

2020

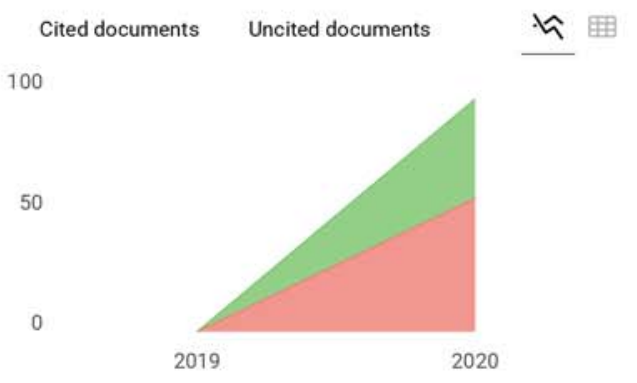

\section{G sClmago Graphica}

Explore, visually communicate and make sense of data with our new free tool.

Get it

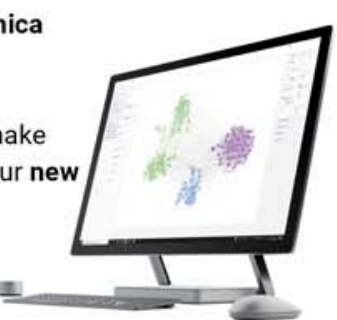

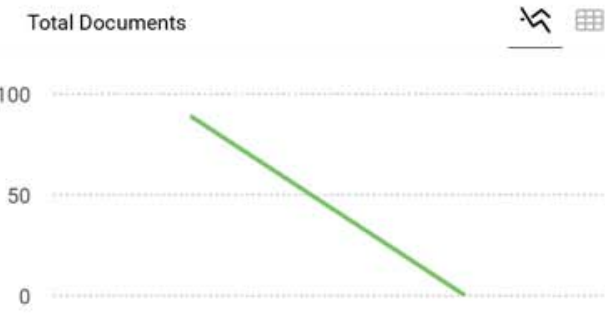

20192020
Citations per document $れ$ 国

0.8

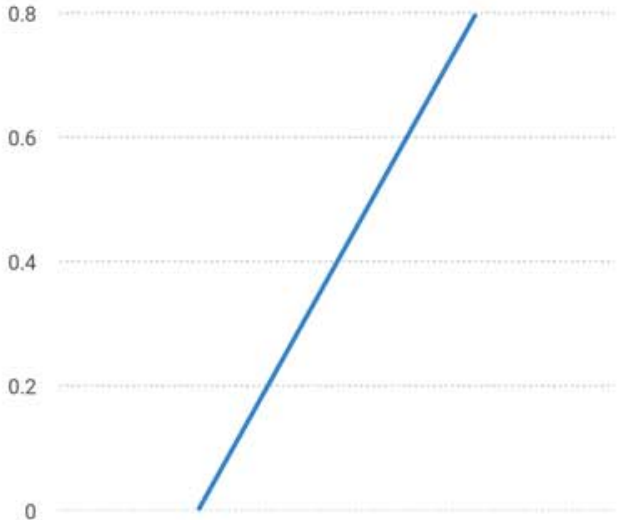

0.2

2019

2020

Cites / Doc. (4 years)

Cites / Doc. (3 years)

- Cites / Doc. (2 years)

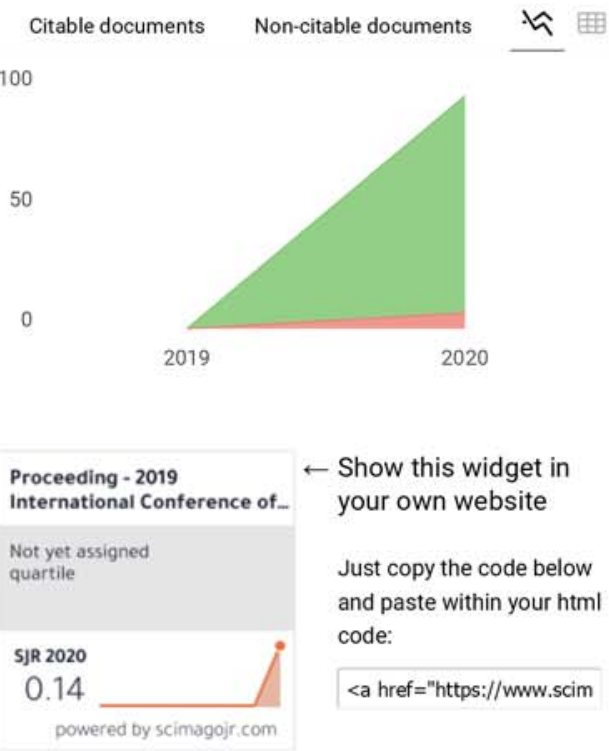

100

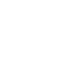

International Conference of__ your own website

Not yet assigne

Just copy the code below and paste within your $\mathrm{html}$

0.14

red by scimagojr.com 


\section{Leave a comment \\ Name \\ Email \\ (will not be published)}

The users of Scimago Journal \& Country Rank have the possibility to dialogue through comments linked to a specific journal. The purpose is to have a forum in which general doubts about the processes of publication in the journal, experiences and other issues derived from the publication of papers are resolved. For topics on particular articles, maintain the dialogue through the usual channels with your editor. 


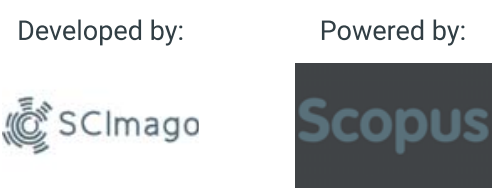

Follow us on @ScimagoJR

Scimago Lab, Copyright 2007-2020. Data Source: Scopus ${ }^{\circledR}$ 


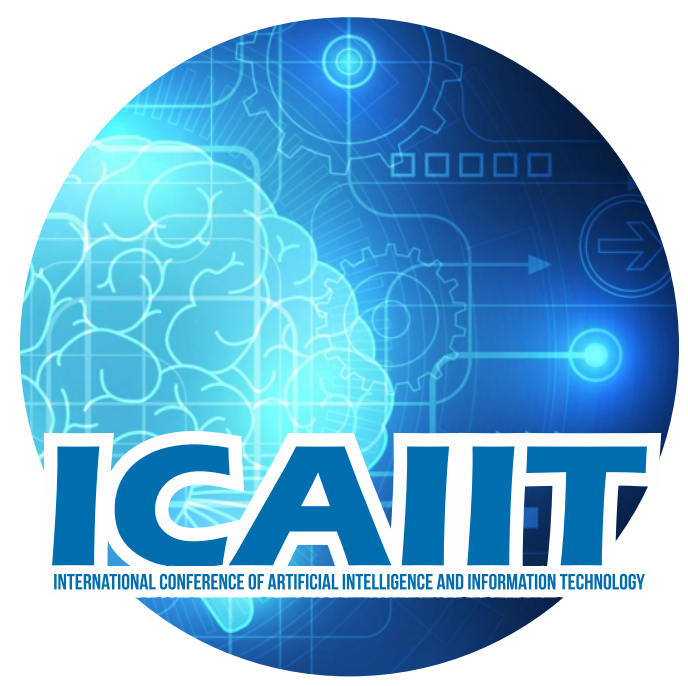

\title{
2019 INTERNATIONAL CONFERENCE OF ARTIFICIAL INTELLIGENCE AND INFORMATION TECHNOLOGY (ICAIIT 2019)
}

\author{
13 - 15 March 2019
}

Platinum Adisutjipto Yogyakarta Hotel \& Conference Center

IEEE Conference Number \#45307

IEEE Catalog Number : CFP19JVE-ART ISBN : 978-1-5386-8448-1 


\section{COPYRIGHTS}

\section{International Conference of Artificial Intelligence and Information Technology}

IEEE Conference Number \#45307

Copyright (@2019 by Universitas Atma Jaya Yogyakarta and Kalbis Institute. All rights reserved

\section{Copyright and Reprint Permission:}

Abstracting is permitted with credit to the source. Libraries are permitted to photocopy beyond the limit of U.S. copyright law for private use of patrons those articles in this volume that carry a code at the bottom of the first page, provided the per-copy fee indicated in the code is paid through Copyright Clearance Center, 222 Rosewood Drive, Danvers, MA 01923. For reprint or republication permission, email to IEEE Copyrights Manager at pubs-permissions@ieee.org. All rights reserved. Copyright (C2019 by IEEE.

IEEE Catalog Number : CFP19JVE-ART

ISBN : : : : :

Additional copies of this publication is available from

IEEE Conference Operations

445 Hoes Lane

Piscataway, NJ 08854 USA

Fax: +17329811769

Email: conference-ops@ieee.org

Availability: Monday-Friday 08:00 - 16:30 Eastern Standard Time

IEEE: Advancing Technology for Humanity 


\section{International Conference of Artificial Intelligence and Information Technology 2019 (ICAIIT) \\ Organizing Committees}

(IEEE International Conference \#45307)

\section{Honorary Chair:}

- Dr. Gregorius Sri Nurhartanto, S.H., LL.M. (Universitas Atma Jaya Yogyakarta)

- Dr. A. Teguh Siswantoro, M.Sc (Universitas Atma Jaya Yogyakarta)

- Dr. Hadi Sutopo (Institut Teknologi dan Bisnis Kalbis)

- Prof. Andrea Corradini (Copenhagen School of Design and Technology, Denmark)

- Associate Professor Wladyslaw Homenda (Warsaw University of Technology, Poland)

- Dr. Suryadiputra Liawatimena (The Indonesia Section Computer Society Chapter, Indonesia)

- Wu Hai Yong, Ph.D.(Nanjing Xiaozhuang University, China)

\section{General Chair:}

- Martinus Maslim (Universitas Atma Jaya Yogyakarta)

- Yulius Denny Prabowo (Institut Teknologi dan Bisnis Kalbis)

\section{Co-Chair:}

- Stephanie Pamela Adithama (Universitas Atma Jaya Yogyakarta)

- Jullend Gatc (Institut Teknologi dan Bisnis Kalbis)

\section{Liaison Chair:}

- Vinindita Citrayasa (Universitas Atma Jaya Yogyakarta)

- Anjar Dwi Astono (Institut Teknologi dan Bisnis Kalbis)

\section{Media and Information Chair:}

- Thomas Adi Purnomo Sidhi (Universitas Atma Jaya Yogyakarta)

- Andreas Hemawan Tri N. (Universitas Atma Jaya Yogyakarta)

\section{Program Chair:}

- B. Yudi Dwiandiyanta (Universitas Atma Jaya Yogyakarta)

- Eduard Rusdianto (Universitas Atma Jaya Yogyakarta)

\section{Publication Chair:}

- Yulius Harjoseputro (Universitas Atma Jaya Yogyakarta)

- Findra Kartika Sari Dewi (Universitas Atma Jaya Yogyakarta)

\section{Program Committee:}

- Agustinus Kris Handoyo (Universitas Atma Jaya Yogyakarta)

- Lucia Misa Indrawati (Universitas Atma Jaya Yogyakarta) 


\section{Technical Committee (reviewers) :}

- Dr. Alb. Joko Santoso (Universitas Atma Jaya Yogyakarta, Indonesia)

- Prof. Suyoto (Universitas Atma Jaya Yogyakarta, Indonesia)

- Dr. Pranowo (Universitas Atma Jaya Yogyakarta, Indonesia)

- Dr. Andi Wahju Rahardjo Emanuel (Universitas Atma Jaya Yogyakarta, Indonesia)

- The Jin Ai, S.T., M.T., Dr.Eng. (Universitas Atma Jaya Yogyakarta, Indonesia)

- Paulus Mudjihartono, S.T., M.T. (Universitas Atma Jaya Yogyakarta, Indonesia)

- Kusworo Anindito, S.T., M.T. (Universitas Atma Jaya Yogyakarta, Indonesia)

- Y. Sigit Purnomo WP., S.T., M.Kom. (Universitas Atma Jaya Yogyakarta, Indonesia)

- Harya Bima Dirgantara S.Kom., M.T.I. (Kalbis Institute, Indonesia)

- Alfa Ryano Yohannis S.T., M.T. (Kalbis Institute, Indonesia)

- Asst. Prof. Thitipong Tanprasert, Ph.D (Assumption University, Thailand)

- Dr. Benjawan Srisura (Assumption University, Thailand)

- Prof. Nanna Suryana Herman (Universiti Teknikal Malaysia Melaka, Malaysia)

- Dr. Jaziar Radianti (University of Agder, Norway)

- I Putu Edy Suardiyana Putra (Macquarie University, Australia)

Thomas Suselo, S.T., M.T. (University of Auckland, Australia)

Ahmed Hussein (University of Mustansiriyah, Iraq)

Shir Mohammad Tavangari (University of Zurich, Switzerland)

Dr. Inggriani Liem (Institut Teknologi Bandung, Indonesia)

Dr. Ridi Ferdiana (Universitas Gadjah Mada Yogyakarta, Indonesia)

Prof. Eko Sediyono (Universitas Kristen Satya Wacana Salatiga, Indonesia)

Dr. Adhi Dharma Wibawa (Institute Teknologi Sepuluh Nopember, Indonesia)

Prof. Dr. Adi Wijaya (Telkom University, Indonesia)

Dr. Mujiono Sadikin (Universitas Mercu Buana, Indonesia)

Ionia Veritawati, S.Si., M.T. (Universitas Pancasila, Indonesia)

Febri Maspiyanti, S.Kom., M.Kom (Universitas Pancasila, Indonesia)

Diah Harnoni Apriyanti, S.T, M.Kom. (Indonesian Institute of Sciences)

Firman Anindra, S.T., MTI (Universitas Nasional, Indonesia)

Erick Fernando, S.Kom., MSI (Bina Nusantara University, Indonesia)

- Surjandy, S.Kom., M.M. (Bina Nusantara University, Indonesia)

Dr. Sudi Mungkasi (Sanata Dharma University, Indonesia)

- Iwan Binanto, S.Si., M.Cs. (Sanata Dharma University, Indonesia)

- Teny Handhayani, S.Kom, M.Kom (Tarumanagara University, Indonesia) 


\section{Table of Contents}

Message from Conference Chair

Welcome Speech by Dr. Suryadiputra Liawatimena as The Indonesia Section Computer

i Society Chapter Chair

Welcome Speech by Dr. A. Teguh Siswantoro as Dean of Industrial Technology Faculty, Universitas Atma Jaya Yogyakarta

Welcome Speech by Dr. Gregorius Sri Nurhartanto, SH. LL.M. as Rector of Universitas Atma iv Jaya Yogyakarta

Welcome Speech by Ir. Sablin Yusuf, M.Sc., M.Comp.Sc. as Rector of Kalbis Institute,

Indonesia

Special Region of Yogyakarta

Universitas Atma Jaya Yogyakarta

KALBIS Institute

Keynote Speaker 1: Professor Andrea Corradini (Copenhagen School of Design and

Technology, Denmark)

Keynote Speaker 2: Associate Professor Władysław Homenda (Warsaw University of

Technology, Poland)

Keynote Speaker 3: HAIYONG WU, Ph. D (Nanjing Xiaozhuang University)

Keynote Speaker 4: Dr. Suryadiputra Liawatimena, S.Kom., PgDip.App.Sci (Universitas Bina

Nusantara, Indonesia)

ICAIIT 2019 Organizing Committees

xxi

Table of Contents

xxiii

Conference Schedule

xxiv

Conference List of Papers

Conference List of Papers per room 
International Conference of Artificial Intelligence and Information Technology 2019 had been listed at IEEE Conference number 45307

(https://conferences.ieee.org/conferences_events/conferences/conferencedetails/45307 ).

Papers in International Conference of Artificial Intelligence and Information Technology 2019 for media communication only which is spread among the authors, Keynote speakers, and other academic colleagues in the International Conference of Artificial Intelligence and Information Technology 2019, at 13-14 March 2019 at Platinum Adisucipto Hotel \& Conference Center, Yogyakarta, Indonesia.

Each paper which is shown in this International Conference of Artificial Intelligence and Information Technology 2019 can be appeared at proceeding of International Conference of Artificial Intelligence and Information Technology 2019 where the authors of each paper should:

1. Present their paper

2. Submit the paper's revision to icaiit.reg@uajy.ac.id Failing to do the requirement will be subjected to eliminated from proceeding of International Conference of Artificial Intelligence and Information Technology 2019.

\section{International Conference of Artificial Intelligence and Information Technology 2019 Conference Schedule}

Wednesday, 13 March 2019

\begin{tabular}{|c|c|c|c|}
\hline Time & \multicolumn{3}{|l|}{ Activity } \\
\hline $08.00-09.00$ & \multicolumn{3}{|c|}{ Participants Registration \& Materials Collection } \\
\hline $09.00-10.00$ & \multicolumn{3}{|c|}{ 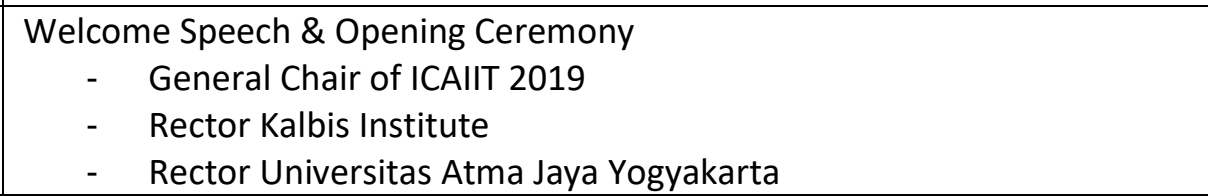 } \\
\hline $10.00-10.30$ & \multicolumn{3}{|l|}{ Coffee Break } \\
\hline $10.30-11.15$ & \multicolumn{3}{|c|}{$\begin{array}{l}\text { Keynote Speech } 1 \\
\text { Professor Andrea Corradini (Copenhagen School of Design and Technology, } \\
\text { Denmark) } \\
\text { Speech Title: Multimodal Data Analysis }\end{array}$} \\
\hline $11.15-12.00$ & \multicolumn{3}{|c|}{$\begin{array}{l}\text { Keynote Speech } 2 \\
\text { HAIYONG WU, Ph. D (School of Information Engineering, Nanjing Xiaozhuang } \\
\text { University) } \\
\text { Speech Title: Deep Learning in Tractography }\end{array}$} \\
\hline $12.00-13.00$ & \multicolumn{3}{|l|}{ Lunch } \\
\hline $\begin{array}{l}\text { 13.00 - } 15.00 \\
\text { (15 Minutes } \\
\text { presentation } \\
\text { per paper) } \\
\text { Session 1: } \\
\text { 1:13.00-13.15 } \\
2: 13.15-13.30 \\
\text { 3:13.30-13.45 } \\
\text { 4:13.45-14.00 } \\
\end{array}$ & $\begin{array}{l}\text { Titanium 1 } \\
1.1570508359 \\
2.1570526507 \\
3.1570526480 \\
4.1570526013 \\
5.1570518440 \\
6.1570519077 \\
7.1570518796 \\
\end{array}$ & \begin{tabular}{|l|} 
Titanium 2 \\
1. 1570512443 \\
2. 1570512569 \\
3. 1570514049 \\
4. 1570507038 \\
5. 1570512457 \\
6. 1570526214 \\
7. 1570513290 \\
\end{tabular} & \begin{tabular}{|l|} 
Titanium $\mathbf{3}$ \\
1. 1570512696 \\
2. 1570512578 \\
3. 1570512583 \\
4. 1570512614 \\
5. 1570512722 \\
6. 1570512735 \\
7. 1570518672 \\
\end{tabular} \\
\hline
\end{tabular}

xxiv 


\begin{tabular}{|c|c|c|c|c|}
\hline $\begin{array}{l}5: 14.00-14.15 \\
6: 14.15-14.30 \\
7: 14.30-14.45\end{array}$ & & & & \\
\hline $15.00-15.30$ & \multicolumn{4}{|l|}{ Coffee Break } \\
\hline $\begin{array}{l}15.30-17.00 \\
\text { (15 Minutes } \\
\text { presentation } \\
\text { per paper) } \\
\text { Session 2: } \\
\text { 1:15.30-15.45 } \\
2: 15.45-16.00 \\
3: 16.00-16.15 \\
4: 16.15-16.30 \\
5: 16.30-16.45 \\
6: 16.45-17.00 \\
\end{array}$ & $\begin{array}{l}\text { Titanium } 1 \\
1.1570515432 \\
2.1570526222 \\
3.1570526304 \\
4.1570526319 \\
5.1570526544 \\
6.1570526452\end{array}$ & $\begin{array}{l}\text { Titanium } 2 \\
1.1570513614 \\
2.1570497900 \\
3.1570497821 \\
4.1570512658 \\
5.1570523975 \\
6.1570525893\end{array}$ & $\begin{array}{l}\text { Titanium } 3 \\
1.1570526455 \\
2.1570526552 \\
3.1570516699 \\
4.1570513732 \\
5.1570509093 \\
6.1570526401\end{array}$ & $\begin{array}{ll}\text { Ballroom } 1 \\
\text { 1. } & 1570521650 \\
\text { 2. } & 1570526538 \\
\text { 3. } & 1570526466 \\
\text { 4. } & 1570526453 \\
\text { 5. } & 1570524081 \\
\text { 6. } & 1570513593\end{array}$ \\
\hline $18.00-20.00$ & \multicolumn{4}{|c|}{ Gala Dinner at Rama Shinta Resto Prambanan Temple } \\
\hline
\end{tabular}

Thursday, 14 March 2019

\begin{tabular}{|c|c|c|c|c|}
\hline Time & \multicolumn{4}{|l|}{ Activity } \\
\hline $08.00-09.00$ & \multicolumn{4}{|c|}{ Registration \& Coffee Break } \\
\hline $09.00-09.45$ & \multicolumn{4}{|c|}{$\begin{array}{l}\text { Keynote Speech } 3 \\
\text { Dr. Suryadiputra Liawatimena, S.Kom., PgDip.App.Sci (Universitas Bina } \\
\text { Nusantara, Indonesia) } \\
\text { Speech Title: Artifical Intelligence in Agriculture \& Fishery }\end{array}$} \\
\hline $09.45-10.30$ & \multicolumn{4}{|c|}{$\begin{array}{l}\text { Keynote Speech 4: } \\
\text { Associate Professor Władysław Homenda (Warsaw University of Technology, } \\
\text { Poland) } \\
\text { Speech Title: Cognitive Maps for Time Series Modeling }\end{array}$} \\
\hline $\begin{array}{l}\text { 10.30 - } 11.30 \\
\text { (15 Minutes } \\
\text { presentation } \\
\text { per paper) } \\
\text { Session 3: } \\
\text { 1:10.30-10.45 } \\
\text { 2:10.45-11.00 } \\
\text { 3:11.00-11.15 } \\
\text { 4:11.15-11.30 }\end{array}$ & $\begin{array}{l}\text { Titanium } 1 \\
1.1570526412 \\
2.1570526525 \\
3.1570516332 \\
4.1570526533\end{array}$ & $\begin{array}{l}\text { Titanium } \mathbf{2} \\
\text { 1. } 1570526555 \\
\text { 2. } 1570526750 \\
\text { 3. } 1570508178 \\
\text { 4. } 1570526557\end{array}$ & $\begin{array}{ll}\text { Ballroom 1 } \\
\text { 1. } 1570526547 \\
\text { 2. } 1570526500 \\
\text { 3. } 1570511011 \\
\text { 4. } 1570522671\end{array}$ & \begin{tabular}{ll}
\multicolumn{2}{c}{ Ballroom 2 } \\
1. & 1570526541 \\
2. & 1570512562 \\
3. & 1570513741 \\
4. & 1570513710
\end{tabular} \\
\hline $11.30-12.30$ & \multicolumn{4}{|l|}{ Lunch } \\
\hline $\begin{array}{l}12.30-15.00 \\
\text { (15 Minutes } \\
\text { presentation } \\
\text { per paper) } \\
\text { Session 4: } \\
\text { 1:12.30-12.45 } \\
2: 12.45-13.00 \\
3: 13.00-13.15 \\
4: 13.15-13.30 \\
5: 13.30-13.45 \\
6: 13.45-14.00 \\
7: 14.00-14.15 \\
8: 14.15-14.30 \\
9: 14.30-14.45 \\
10: 14.45-15.00 \\
\end{array}$ & $\begin{array}{l}\text { Titanium 1 } \\
1.1570526563 \\
2.1570516556 \\
3.1570523658 \\
4.1570524898 \\
5.1570524566 \\
6.1570526512 \\
7.1570484576 \\
8.1570511144 \\
9.1570520869\end{array}$ & $\begin{array}{l}\text { Titanium } 2 \\
\text { 1. } 1570512995 \\
\text { 2. } 1570510797 \\
\text { 3. } 1570510791 \\
\text { 4. } 1570526499 \\
\text { 5. } 1570526192 \\
\text { 6. } 1570526423 \\
\text { 7. } 1570523474 \\
\text { 8. } 1570526180 \\
\text { 9. } 1570514074\end{array}$ & $\begin{array}{ll}\text { Ballroom } 1 \\
\text { 1. } & 1570512694 \\
\text { 2. } & 1570497875 \\
\text { 3. } & 1570512201 \\
\text { 4. } & 1570513608 \\
\text { 5. } & 1570513712 \\
\text { 6. } & 1570513896 \\
\text { 7. } & 1570513597 \\
\text { 8. } & 1570526714 \\
\text { 9. } & 1570526573\end{array}$ & $\begin{array}{ll}\text { Ballroom 2 } \\
\text { 1. } & 1570515288 \\
\text { 2. } & 1570523931 \\
\text { 3. } & 1570526385 \\
\text { 4. } & 1570526502 \\
\text { 5. } & 1570526281 \\
\text { 6. } & 1570526529 \\
\text { 7. } & 1570521672 \\
\text { 8. } & 1570526218 \\
\text { 9. } & 1570526456 \\
\text { 10. } & 1570526549\end{array}$ \\
\hline $15.00-15.30$ & \multicolumn{4}{|l|}{ Coffee Break } \\
\hline
\end{tabular}




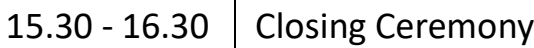

\section{Friday, 15 March 2019}

\begin{tabular}{|c|l|}
\hline Time & Activity \\
\hline $07.00-20.00$ & Excursion (for Full Package only) \\
\hline
\end{tabular}

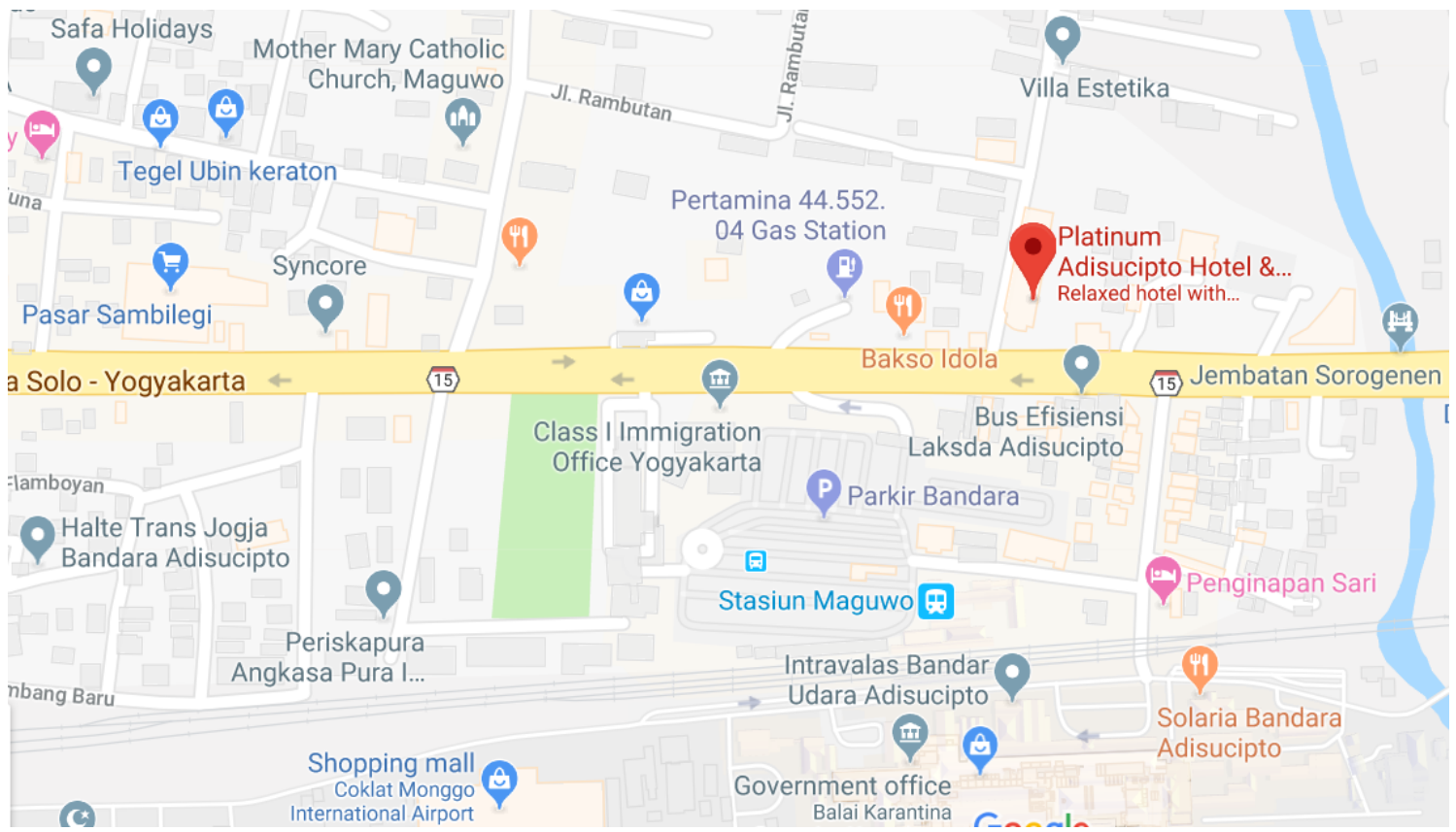




\section{International Conference of Artificial Intelligence and Information Technology 2019 \\ List of Papers}

\begin{tabular}{|c|c|c|c|}
\hline $\begin{array}{l}\text { Paper } \\
\text { Code }\end{array}$ & Paper Title (Authors) & $\begin{array}{c}\text { Room } \\
\text { (Session) Time }\end{array}$ & Page \\
\hline 1570484576 & $\begin{array}{l}\text { A Comparative Study on Variational Autoencoders and Generative } \\
\text { Adversarial Networks } \\
\text { (Mirza Sami, Iftekharul Mobin) }\end{array}$ & $\begin{array}{l}\text { Titanium } 1(4), 14.00- \\
14.15\end{array}$ & 1 \\
\hline 1570497821 & $\begin{array}{l}\text { Face Detection using Haar Cascades to Filter Selfie Face Image on Instagram } \\
\text { (Adri Priadana, Muhammad Habibi) }\end{array}$ & $\begin{array}{l}\text { Titanium } 2(2), 16.00- \\
16.15\end{array}$ & 6 \\
\hline 1570497875 & $\begin{array}{l}\text { MREAK : Morphological Retina Keypoint Descriptor } \\
\text { (Himanshu Vaghela, Manan Oza, Sudhir Bagul) }\end{array}$ & $\begin{array}{l}\text { Ballroom } 1(4), 12.45- \\
13.00\end{array}$ & 10 \\
\hline 1570497900 & $\begin{array}{l}\text { Semi-Supervised Image-to-Image Translation } \\
\text { (Manan Oza, Himanshu Vaghela, Sudhir Bagul) }\end{array}$ & $\begin{array}{l}\text { Titanium } 2(2), 15.45- \\
16.00\end{array}$ & 16 \\
\hline 1570507038 & $\begin{array}{l}\text { Social Media Prototyping for Web-based Property Business } \\
\text { (Harya Bima Dirgantara, Paramaresthi Windriyani, Rendy Adiwikarta) }\end{array}$ & $\begin{array}{l}\text { Titanum } 2(1), 13.45- \\
14.00\end{array}$ & 21 \\
\hline 1570508178 & $\begin{array}{l}\text { Simple Implementation of Fuzzy Controller for Low Cost Microcontroller } \\
\text { (Wakhyu Dwiono, Arif Johar Taufiq, W Winarso) }\end{array}$ & $\begin{array}{l}\text { Titanium } 2 \text { (3), 11.00- } \\
11.15\end{array}$ & 26 \\
\hline 1570508359 & $\begin{array}{l}\text { Design of Manufacture Professional Training and Assessment Information } \\
\text { System in The Implementation of PBET (Production Based Education and } \\
\text { Training) Learning Activity Model } \\
\text { (Yustina Tritularsih) }\end{array}$ & $\begin{array}{l}\text { Titanium } 1(1), 13.00- \\
13.15\end{array}$ & 31 \\
\hline 1570509093 & $\begin{array}{l}\text { Extraction of Skull and Face Surfaces from CT Images } \\
\text { (Masy Ari Ulinuha, Eko Mulyanto Yuniarno, I Ketut Eddy Purnama, } \\
\text { Mochamad Hariadi) }\end{array}$ & $\begin{array}{l}\text { Titanium } 3(2), 16.30- \\
16.45\end{array}$ & 37 \\
\hline 1570510791 & $\begin{array}{l}\text { Real-time Moving Object Video Tracking using Support Vector Machines for } \\
\text { Visual Servo Application } \\
\text { (Modestus Oliver Asali, Saripudin, Bambang Trilaksono, Toto Indriyanto) }\end{array}$ & $\begin{array}{l}\text { Titanium } 2(4), 13.00- \\
13.15\end{array}$ & 41 \\
\hline 1570510797 & $\begin{array}{l}\text { Visual Servoing using Mixed Sensitivity Ho Control for Yaw-Pitch Camera } \\
\text { Platform } \\
\text { (Saripudin, Modestus Oliver Asali, Bambang Riyanto Trilaksono, Toto } \\
\text { Indriyanto) }\end{array}$ & $\begin{array}{l}\text { Titanium } 2(4), 12.45- \\
13.00\end{array}$ & 48 \\
\hline 1570511011 & $\begin{array}{l}\text { Automatic Lecture Video Content Summarization with Attention-based } \\
\text { Recurrent Neural Network } \\
\text { (Muhammad Bagus Andra, Tsuyoshi Usagawa) }\end{array}$ & $\begin{array}{l}\text { Ballroom 1 (3), 11.00- } \\
11.15\end{array}$ & 54 \\
\hline 1570511144 & $\begin{array}{l}\text { Design and Development Meeting Schedule Management Application using } \\
\text { the RAD Method } \\
\text { (Egia Rosi Subhiyakto, Yani Parti Astuti) }\end{array}$ & $\begin{array}{l}\text { Titanium } 1(4), 14.15- \\
14.30\end{array}$ & 60 \\
\hline 1570512201 & $\begin{array}{l}\text { The Influence of Sampling Frequency on Guitar Chord Recognition using DST } \\
\text { Based Segment Averaging } \\
\text { (Linggo Sumarno) }\end{array}$ & $\begin{array}{l}\text { Ballroom } 1(4), 13.00- \\
13.15\end{array}$ & 65 \\
\hline 1570512443 & $\begin{array}{l}\text { The Priority of Tourism Destinations Development using 6AsTD Framework } \\
\text { and TOPSIS } \\
\text { (Yunifa Miftachul Arif, Supeno Mardi Susiki Nugroho, Mochamad Hariadi) }\end{array}$ & $\begin{array}{l}\text { Titanium } 2(1), 13.00- \\
13.15\end{array}$ & 70 \\
\hline 1570512457 & $\begin{array}{l}\text { Systematic Literature Review of Profiling Analysis Based on Social Media } \\
\text { (Mihuandayani, Ema Utami, Anggit Dwi Hartanto, Sumarni Adi, Suwanto } \\
\text { Raharjo) }\end{array}$ & $\begin{array}{l}\text { Titanium } 2(1), 14.00- \\
14.15\end{array}$ & 77 \\
\hline 1570512562 & $\begin{array}{l}\text { NDNization of IP Network Based On Communication Flow Model } \\
\text { (Fandhy Bayu Rukmana, Nina Hendrarini, Riri Fitri Sari) }\end{array}$ & $\begin{array}{l}\text { Ballroom } 2(3), 10.45- \\
11.00\end{array}$ & 83 \\
\hline 1570512569 & $\begin{array}{l}\text { Improve Smart Waste Management to Preserve Tourist Attractions } \\
\text { Yogyakarta in loT Environment } \\
\text { (RANIA RIZKI ARINTA, Dominikus Boli Watomakin, Suyoto) }\end{array}$ & $\begin{array}{l}\text { Titanium } 2 \text { (1), 13.15- } \\
13.30\end{array}$ & 88 \\
\hline 1570512578 & $\begin{array}{l}\text { Smart Kost: Ubiquitous Boarding House Controlling and Monitoring System } \\
\text { in Industry } 4.0 \\
\text { (Julius Galih Prima Negara, Alfredo Gormantara, Suyoto) }\end{array}$ & $\begin{array}{l}\text { Titanium } 3(1), 13.15- \\
13.30\end{array}$ & 94 \\
\hline 1570512583 & IoT Based: Improving Control System For High-Quality Beef in Supermarkets & $\begin{array}{l}\text { Titanium } 3(1), 13.30- \\
13.45\end{array}$ & 99 \\
\hline
\end{tabular}




\begin{tabular}{|c|c|c|c|}
\hline & (BALTRA AGUSTI PRAMAJURI, Erni Widarti, Suyoto) & & \\
\hline 1570512614 & $\begin{array}{l}\text { IOT: Improved Home Energy Control System Based on Consumer Behavior } \\
\text { (Melky Radja, Gilbert Gutabaga Hungilo, Gahizi Emmanuel, Suyoto) }\end{array}$ & $\begin{array}{l}\text { Titanium } 3(1), 13.45- \\
14.00\end{array}$ & 104 \\
\hline 1570512658 & $\begin{array}{l}\text { A classification approach of emotional reactions while driving a vehicle } \\
\text { (Andrea Corradini, Alexander Efa) }\end{array}$ & $\begin{array}{l}\text { Titanium } 2(2), 16.15- \\
16.30\end{array}$ & 109 \\
\hline 1570512694 & $\begin{array}{l}\text { Determining the Neural Network Topology from the Viewpoint of Kuhn's } \\
\text { Philosophy and Popper's Philosophy } \\
\text { (MUHAMMAD IBNU CHOLDUN RACHMATULLAH, Kridanto Surendro, Judhi } \\
\text { Santoso, Dimitri Mahayana) }\end{array}$ & $\begin{array}{l}\text { Ballroom } 1(4), 12.30- \\
12.45\end{array}$ & 115 \\
\hline 1570512696 & $\begin{array}{l}\text { IoT-Based Smart And Healthy Wardrobe System } \\
\text { (Fedelis Brian Putra Prakasa, Jaouja Maiga, Suyoto) }\end{array}$ & $\begin{array}{l}\text { Titanium } 3(1), 13.00- \\
13.15\end{array}$ & 119 \\
\hline 1570512722 & $\begin{array}{l}\text { loT Based: Hydroponic Using Drip Non-Circulation System for Paprika } \\
\text { (Dhana Sudana, Dadang Eman, Suyoto) }\end{array}$ & $\begin{array}{l}\text { Titanium } 3(1), 14.00- \\
14.15\end{array}$ & 124 \\
\hline 1570512735 & $\begin{array}{l}\text { Smart hydroponic farming with loT-based climate and nutrient manipulation } \\
\text { system } \\
\text { (Rangga Perwiratama, Yosef Kelly Setiadi, Suyoto) }\end{array}$ & $\begin{array}{l}\text { Titanium } 3(1), 14.15- \\
14.30\end{array}$ & 129 \\
\hline 1570512995 & $\begin{array}{l}\text { Audio Steganography Using Lifting Wavelet Transform and Dynamic Key } \\
\text { (Mohamad Anwar, Moechammad Sarosa, Erfan Rohadi) }\end{array}$ & $\begin{array}{l}\text { Titanium } 2(4), 12.30- \\
12.45\end{array}$ & 133 \\
\hline 1570513290 & $\begin{array}{l}\text { Automated Test Suite for Regression Testing Based on Serenity Framework: } \\
\text { A Case Study } \\
\text { (Fransiskus Anindita Kristiawan Pramana Gentur Sutapa, Sri Suning } \\
\text { Kusumawardani, Adhistya Erna Permanasari) }\end{array}$ & $\begin{array}{l}\text { Titanium } 2(1), 14.30- \\
14.45\end{array}$ & 138 \\
\hline 1570513593 & $\begin{array}{l}\text { Clutter Mitigation Technique on OFDM MIMO Radar } \\
\text { (Risdilah Mimma Untsa, Gamantyo Hendrantoro, Puji Handayani) }\end{array}$ & $\begin{array}{l}\text { Ballroom } 1 \text { (2), 16.45- } \\
17.00\end{array}$ & 145 \\
\hline 1570513597 & $\begin{array}{l}\text { Switching Formation and Topology in Cooperative Multi-Agent Source } \\
\text { Seeking Using Gradient Estimation } \\
\text { (MOCHAMMAD SAHAL, Trihastuti Agustinah, Achmad Jazidie) }\end{array}$ & $\begin{array}{l}\text { Ballroom } 1 \text { (4), 14.00- } \\
14.15\end{array}$ & 151 \\
\hline 1570513608 & $\begin{array}{l}\text { Forming Formation of Particle Swarm using Artificial Neural Network Self } \\
\text { Organizing Map (ANN-SOM) with 2-leveled Strategy } \\
\text { (Bayu Fandidarma, Achmad Jazidie, Rusdhianto Efendi Abdul Kadir) }\end{array}$ & $\begin{array}{l}\text { Ballroom } 1(4), 13.15- \\
13.30\end{array}$ & 157 \\
\hline 1570513614 & $\begin{array}{l}\text { Complexity Reduction for Multiview HEVC Codec Using FPGA } \\
\text { (M. Suhairi, Wirawan, Endroyono, Astria Nur Irfansyah) }\end{array}$ & $\begin{array}{l}\text { Titanium } 2(2), 15.30- \\
15.45\end{array}$ & 163 \\
\hline 1570513710 & $\begin{array}{l}\text { Trajectory Tracking Automated Guided Vehicle Using Fuzzy Controller } \\
\text { (Mamat Septyan, Trihastuti Agustinah) }\end{array}$ & $\begin{array}{l}\text { Ballroom } 2(3), 11.15- \\
11.30\end{array}$ & 169 \\
\hline 1570513712 & $\begin{array}{l}\text { Modified Ant Colony Algorithm For Swarm Multi Agent Exploration on } \\
\text { Target Searching in Unknown Environment } \\
\text { (Yoan Purbolingga, Achmad Jazidie, Rusdhianto Efendi Abdul Kadir) }\end{array}$ & $\begin{array}{l}\text { Ballroom } 1(4), 13.30- \\
13.45\end{array}$ & 175 \\
\hline 1570513732 & $\begin{array}{l}\text { EEG-based Mental Fatigue Detection Using Cognitive Tests and RVM } \\
\text { Classification } \\
\text { (Andi Setiawan, Adhi Dharma Wibawa, Evi Septiana Pane, Mauridhi Hery } \\
\text { Purnomo) }\end{array}$ & $\begin{array}{l}\text { Titanium } 3(2), 16.15- \\
16.30\end{array}$ & 180 \\
\hline 1570513741 & $\begin{array}{l}\text { REINFORCEMENT POINT AND FUZZY INPUT DESIGN OF FUZZY Q-LEARNING } \\
\text { FOR MOBILE ROBOT NAVIGATION SYSTEM } \\
\text { (Arga Dwi Pambudi, Trihastuti Agustinah, Rusdhianto Efendi Abdul Kadir) }\end{array}$ & $\begin{array}{l}\text { Ballroom } 2(3), 11.00- \\
11.15\end{array}$ & 186 \\
\hline 1570513896 & $\begin{array}{l}\text { Parameter Identifiability of Phased-MIMO Radar } \\
\text { (Muttaqin Hardiwansyah, Syahfrizal Tahcfulloh, Gamantyo Hendrantoro) }\end{array}$ & $\begin{array}{l}\text { Ballroom } 1(4), 13.45- \\
14.00\end{array}$ & 192 \\
\hline 1570514049 & $\begin{array}{l}\text { Review of Benefit Using Gamification Element for Countryside Tourism } \\
\text { (Fedelis Brian Putra Prakasa, Andi Wahju Rahardjo Emanuel) }\end{array}$ & $\begin{array}{l}\text { Titanium } 2(1), 13.30- \\
13.45\end{array}$ & 196 \\
\hline 1570514074 & $\begin{array}{l}\text { The Classification of the Movie Genre based on Synopsis of the Indonesian } \\
\text { Film } \\
\text { (Antonius Christiyanto Saputra, Pius Guiseppe Sarto Aji Tetuko, Giovani } \\
\text { Christian Nugroho, Anjelina Br Sitepu, Stanley, Yohanes Sigit Purnomo WP) }\end{array}$ & $\begin{array}{l}\text { Titanium } 2(4), 14.30- \\
14.45\end{array}$ & 201 \\
\hline 1570515288 & $\begin{array}{l}\text { A Study of Text Classification for Indonesian News Article } \\
\text { (Grelly Lucia Yovellia Londo, Dwiky Hutomo Kartawijaya, Muhammad Rafi } \\
\text { Aryasuta P, Hesti Tri Ivariyani, Dipo Ariyandi, Yohanes Sigit Purnomo WP) }\end{array}$ & $\begin{array}{l}\text { Ballroom } 2(4), 12.30- \\
12.45\end{array}$ & 205 \\
\hline 1570515432 & $\begin{array}{l}\text { Big Data Analytics: Estimation of Destination for Users of Bus Rapid Transit } \\
\text { (BRT) Public Transportation in Jakarta } \\
\text { (MUHAMMAD SYARIF, Widyawan, Teguh Bharata Adji) }\end{array}$ & $\begin{array}{l}\text { Titanium } 1(2), 15.30- \\
15.45\end{array}$ & 209 \\
\hline 1570516332 & $\begin{array}{l}\text { Gamification of Mobile-based Japanese Language Shadowing } \\
\text { (Hans Christian Kurniawan, Benhard Sitohang, Satrio Adi Rukmono) }\end{array}$ & $\begin{array}{l}\text { Titanium } 1 \text { (3), 11.00- } \\
11.15\end{array}$ & 215 \\
\hline
\end{tabular}




\begin{tabular}{|c|c|c|c|}
\hline 1570516556 & $\begin{array}{l}\text { A Preliminary Performance Evaluation of Population-Based Algorithms in } \\
\text { VANET } \\
\text { (Ronald Adrian, Selo Sulistyo, I Wayan Mustika, Sahirul Alam) }\end{array}$ & $\begin{array}{l}\text { Titanium } 1 \text { (4), } 12.45- \\
13.00\end{array}$ & 220 \\
\hline 1570516699 & $\begin{array}{l}\text { Classification of Premature Ventricular Contraction based on ECG Signal } \\
\text { using Multiorder Rényi Entropy } \\
\text { (Achmad Rizal, Inung Wijayanto) }\end{array}$ & $\begin{array}{l}\text { Titanium } 3(2), 16.00- \\
16.15\end{array}$ & 225 \\
\hline 1570518440 & $\begin{array}{l}\text { Predictive Analytics for Predicting Customer Behavior } \\
\text { (Asniar, Kridanto Surendro) }\end{array}$ & $\begin{array}{l}\text { Titanium } 1(1), 14.00- \\
14.15\end{array}$ & 230 \\
\hline 1570518672 & $\begin{array}{l}\text { Internet of Things: Roboboat for Water Area Monitoring using 4G network } \\
\text { and Google Firebase } \\
\text { (Dadan Nur Ramadan, Sugondo Hadiyoso, Ahmad Rizaldi Sakti) }\end{array}$ & $\begin{array}{l}\text { Titanium } 3(1), 14.30- \\
14.45\end{array}$ & 234 \\
\hline 1570518796 & $\begin{array}{l}\text { Decision Support Systems to Determining Programme for Students Using } \\
\text { DBSCAN And Naive Bayes } \\
\text { (Erna Daniati) }\end{array}$ & $\begin{array}{l}\text { Titanium } 1(1), 14.30- \\
14.45\end{array}$ & 238 \\
\hline 1570519077 & $\begin{array}{l}\text { Fluid Simulation Based on Material Point Method with Neural Network } \\
\text { (Pandu Akbar Dwikatama, Dody Dharma, Achmad Imam Kistijantoro) }\end{array}$ & $\begin{array}{l}\text { Titanium } 1(1), 14.15- \\
14.30\end{array}$ & 244 \\
\hline 1570520869 & $\begin{array}{l}\text { Designing IT-based Skills and Competency Learning System } \\
\text { (Sigit Triyono, Yetti Supriyati, Billy Tunas) }\end{array}$ & $\begin{array}{l}\text { Titanium } 1(4), 14.30- \\
14.45\end{array}$ & 250 \\
\hline 1570521650 & $\begin{array}{l}\text { Determining the Threshold Value for Identification of the Goblet Cells in } \\
\text { Chicken Small Intestine } \\
\text { (Dedi Sepriana, Kusworo Adi, Catur Edi Widodo) }\end{array}$ & $\begin{array}{l}\text { Ballroom } 1(2), 15.30- \\
15.45\end{array}$ & 255 \\
\hline 1570521672 & $\begin{array}{l}\text { Information Retrieval System for Searching JSON Files with Vector Space } \\
\text { Model Method } \\
\text { (Eko Wahyudi, Sfenrianto, M Jundi Hakim, Okky Robiana Sulaeman, } \\
\text { Rochmat Setiyawan, Reko Subandi) }\end{array}$ & $\begin{array}{l}\text { Ballroom } 2(4), 14.00- \\
14.15\end{array}$ & 260 \\
\hline 1570522671 & $\begin{array}{l}\text { Traffic Sign Image Recognition Using Gabor Wavelet and Principle } \\
\text { Component Analysis } \\
\text { (Immawan Wicaksono, Hendra Kusuma, Tri Arief Sardjono) }\end{array}$ & $\begin{array}{l}\text { Ballroom } 1 \text { (3), 11.15- } \\
11.30\end{array}$ & 266 \\
\hline 1570523474 & $\begin{array}{l}\text { COMPARISON OF DISTANCE METHODS IN K-MEANS ALGORITHM FOR } \\
\text { DETERMINING VILLAGE STATUS IN BEKASI DISTRICT } \\
\text { (Yoga Religia, Aswan Supriyadi Sunge) }\end{array}$ & $\begin{array}{l}\text { Titanium } 2(4), 14.00- \\
14.15\end{array}$ & 270 \\
\hline 1570523658 & $\begin{array}{l}\text { Rough-Regression for Categorical Data Prediction based on Case Study } \\
\text { (Riswan Efendi, Susnaningsih Mu'at, Voni Apriana Dewi, Nelsi Arisandy, } \\
\text { Noor Azah Samsudin, Dadang Syarif Sihabudin Sahid) }\end{array}$ & $\begin{array}{l}\text { Titanium } 1(4), 13.00- \\
13.15\end{array}$ & 277 \\
\hline 1570523931 & $\begin{array}{l}\text { A Comparison of the Use of Several Different Resources on Lexicon Based } \\
\text { Indonesian Sentimentc Analysis on App Review Dataset } \\
\text { (Bayu Trisna Pratama, Ema Utami, Andi Sunyoto) }\end{array}$ & $\begin{array}{l}\text { Ballroom } 2(4), 12.45- \\
13.00\end{array}$ & 282 \\
\hline 1570523975 & $\begin{array}{l}\text { Image Based Leaf Area Measurement Method Using Artificial Neural } \\
\text { Network } \\
\text { (Joko Siswantoro, Ida Bagus Made Artadana) }\end{array}$ & $\begin{array}{l}\text { Titanium } 2(2), 16.30- \\
16.45\end{array}$ & 288 \\
\hline 1570524081 & $\begin{array}{l}\text { Contrast-enhanced Based on Abdominal Kernels for CT Image Noise } \\
\text { Reduction } \\
\text { (Riky Tri Yunardi, Qurratul Istiqomah, Risalatul Latifah) }\end{array}$ & $\begin{array}{l}\text { Ballroom } 1(2), 16.30- \\
16.45\end{array}$ & 293 \\
\hline 1570524566 & $\begin{array}{l}\text { Improved Particle Swarm Optimization By Fast Simulated Annealing } \\
\text { Algorithm } \\
\text { (Samar Salem Ahmed Omar Bashath, Amelia Ritahani Ismail) }\end{array}$ & $\begin{array}{l}\text { Titanium } 1(4), 13.30- \\
13.45\end{array}$ & 297 \\
\hline 1570524898 & $\begin{array}{l}\text { FHC-Optimization Model for Deciding the Objective Hajj Pilgrims to } \\
\text { Restricted Quota (Case Study: Hajj Pilgrimage Procedure in Indonesia) } \\
\text { (Ditdit Nugeraha Utama, Muhammad Faturrahman, Methamazid Rusdi, } \\
\text { Ibnu Yahya Saputra, Fuji Suci Isnaeni, Bayu Waspodo) }\end{array}$ & $\begin{array}{l}\text { Titanium } 1(4), 13.15- \\
13.30\end{array}$ & 302 \\
\hline 1570525893 & $\begin{array}{l}\text { Detection of Anomalies in Citrus Leaves Using Digital Image Processing and } \\
\mathrm{T}^{2} \text { Hotelling Multivariate Control Chart } \\
\text { (Marcelinus Alfasisurya Setya Adhiwibawa, Waego Nugroho, Solimun) }\end{array}$ & $\begin{array}{l}\text { Titanium } 2(2), 16.45- \\
17.00\end{array}$ & 310 \\
\hline 1570526013 & $\begin{array}{l}\text { Adapted Flower Pollination Algorithm for Lecturer-Class Assignment } \\
\text { (MA. SHIELA C. SAPUL, Rachsuda Setthawong, Pisal Setthawong) }\end{array}$ & $\begin{array}{l}\text { Titanium } 1(1), 13.45- \\
14.00\end{array}$ & 315 \\
\hline 1570526180 & $\begin{array}{l}\text { Fuzzy Coordinator based Al for Dynamic Difficulty Adjustment in Starcraft } 2 \\
\text { (Muhammad Daryl Bey Sandy Supriyadi, Supeno Mardi Susiki Nugroho, } \\
\text { Mochamad Hariadi) }\end{array}$ & $\begin{array}{l}\text { Titanum } 2(4), 14.15- \\
14.30\end{array}$ & 322 \\
\hline 1570526192 & $\begin{array}{l}\text { Digital Overcurrent Relay Implementation With Non-Standard Inverse Curve } \\
\text { Modelling Using Adaptive Neuro Fuzzy Inference System }\end{array}$ & $\begin{array}{l}\text { Titanium } 2 \text { (4), 13.30- } \\
13.45\end{array}$ & 327 \\
\hline
\end{tabular}




\begin{tabular}{|c|c|c|c|}
\hline & $\begin{array}{l}\text { (Dimas Okky Anggriawan, Eka Prasetyono, Fikri Fahrisi, Anang Budikarso, } \\
\text { Anang Tjahjono, Hardefa Rizky Putu Rogonondo) }\end{array}$ & & \\
\hline 1570526214 & $\begin{array}{l}\text { Harmonics Reduction for Four-Leg Distribution Network-Connected Single } \\
\text { Phase Transformerless PV Inverter System Using Diagonal Recurrent Neural } \\
\text { Network } \\
\text { (Dedy Kurnia Setiawan, Mochamad Ashari, Heri Suryoatmojo) }\end{array}$ & $\begin{array}{l}\text { Titanium } 2(1), 14.15- \\
14.30\end{array}$ & 331 \\
\hline 1570526218 & $\begin{array}{l}\text { Melanoma Classification Using Texture and Wavelet Analysis } \\
\text { (Akhiyar Waladi, Nanda Maulina Firdaus, Aniati M. Arymurthy) }\end{array}$ & $\begin{array}{l}\text { Ballroom } 2(4), 14.15- \\
14.30\end{array}$ & 336 \\
\hline 1570526222 & $\begin{array}{l}\text { Deep Learning-Based Patient Visits Forecasting Using Long Short Term } \\
\text { Memory } \\
\text { (Hayuning Titi Karsanti, Igi Ardiyanto, Lukito Edi Nugroho) }\end{array}$ & $\begin{array}{l}\text { Titanium } 1(2), 15.45- \\
16.00\end{array}$ & 344 \\
\hline 1570526281 & $\begin{array}{l}\text { Offensive Language Detection using Artificial Neural Network } \\
\text { (Meredita Susanty, Ahmad Fauzan Rahman, Muhammad Dzaky } \\
\text { Normansyah, Ade Irawan, Sahrul) }\end{array}$ & $\begin{array}{l}\text { Ballroom } 2 \text { (4), } 13.30- \\
13.45\end{array}$ & 350 \\
\hline 1570526304 & $\begin{array}{l}\text { Customizable Dynamic Hand Gesture recognition System for Motor } \\
\text { Impaired people using Siamese neural network } \\
\text { (Pullakandam Muralidhar, Prashanth Sateesh, Amartya Saha) }\end{array}$ & $\begin{array}{l}\text { Titanium } 1(2), 16.00- \\
16.15\end{array}$ & 354 \\
\hline 1570526319 & $\begin{array}{l}\text { Use of ARIMA Method To Predict The Number of Train Passenger In Malang } \\
\text { City } \\
\text { (Triyanna Widiyaningtyas, Muladi, Adiba Qonita) }\end{array}$ & $\begin{array}{l}\text { Titanium } 1(2), 16.15- \\
16.30\end{array}$ & 359 \\
\hline 1570526385 & $\begin{array}{l}\text { Sentiment Analysis In Twitter Using Lexicon Based and Polarity } \\
\text { Multiplication } \\
\text { (Kusrini, Mochamad Mashuri) }\end{array}$ & $\begin{array}{l}\text { Ballroom } 2(4), 13.00- \\
13.15\end{array}$ & 365 \\
\hline 1570526401 & $\begin{array}{l}\text { Heart Rate Estimation from Wrist-Type Photoplethysmographic Signals } \\
\text { Corrupted by Intense Motion Artifacts using NLMS Adaptive Filter and } \\
\text { Spectral Peak Tracking } \\
\text { (Put Gani Ayub Tamudia, Astri Handayani, Agung Wahyu Setiawan) }\end{array}$ & $\begin{array}{l}\text { Titanium } 3(2), 16.45- \\
17.00\end{array}$ & 369 \\
\hline 1570526412 & $\begin{array}{l}\text { The Effect of Game Experience from Counter-Strike: Global Offensive } \\
\text { (Sasmoko Sasmoko, Jason Harsono, Yogi Udjaja, Yasinta Indrianti, Jurike } \\
\text { Moniaga) }\end{array}$ & $\begin{array}{l}\text { Titanium } 1(3), 10.30- \\
10.45\end{array}$ & 374 \\
\hline 1570526423 & $\begin{array}{l}\text { Dynamic Background Video Forgery Detection using Gaussian Mixture } \\
\text { Model } \\
\text { (Nugroho Satriyanto, Rinaldi Munir, Harlili) }\end{array}$ & $\begin{array}{l}\text { Titanium } 2(4), 13.45- \\
14.00\end{array}$ & 379 \\
\hline 1570526452 & $\begin{array}{l}\text { Study on a Train- and Bus-based Delay-Tolerant Networks: Scheduled } \\
\text { Mobility and Impact on Routing } \\
\text { (Agus Urip Ari Wibowo, Selo Sulistyo, I Wayan Mustika) }\end{array}$ & $\begin{array}{l}\text { Titanium } 1(2), 16.45- \\
17.00\end{array}$ & 384 \\
\hline 1570526453 & $\begin{array}{l}\text { A Study on Part Affinity Fields Implementation for Human Pose Estimation } \\
\text { with Deep Neural Network } \\
\text { (Jessika, Isca Amanda, Hasna Marhamah Auliya, Astri Handayani) }\end{array}$ & $\begin{array}{l}\text { Ballroom } 1 \text { (2), 16.15- } \\
16.30\end{array}$ & 391 \\
\hline 1570526455 & $\begin{array}{l}\text { Automated Segmentation of Breast Tissue and Pectoral Muscle in Digital } \\
\text { Mammography } \\
\text { (Aulia Rahmatika, Astri Handayani, Agung Wahyu Setiawan) }\end{array}$ & $\begin{array}{l}\text { Titanium } 3(2), 15.30- \\
15.45\end{array}$ & 397 \\
\hline 1570526456 & $\begin{array}{l}\text { Proportional-Derivative Control for Quadrotor Stabilization under Inertia } \\
\text { Perturbation } \\
\text { (Nurman Setiawan, Samiadji Herdjunanto, Adha Imam Cahyadi) }\end{array}$ & $\begin{array}{l}\text { Ballroom } 2 \text { (4), } 14.30- \\
14.45\end{array}$ & 402 \\
\hline 1570526466 & $\begin{array}{l}\text { Determining Banana Types and Ripeness from Image using Machine } \\
\text { Learning Methods } \\
\text { (Irzal Ahmad Sabilla, Cahyaningtyas Sekar Wahyuni, Chastine Fatichah, Darlis } \\
\text { Herumurti) }\end{array}$ & $\begin{array}{l}\text { Ballroom } 1(2), 16.00- \\
16.15\end{array}$ & 407 \\
\hline 1570526480 & $\begin{array}{l}\text { Predicting Candidates For Fit And Proper Test Using K-Nearest Neighbor } \\
\text { (TIO SAMPURNO, Beni Hediyantama, Martini Ayu Widiyati }\end{array}$ & $\begin{array}{l}\text { Titanium } 1(1), 13.30- \\
13.45\end{array}$ & 413 \\
\hline 1570526499 & $\begin{array}{l}\text { Face Recognition of Low-Resolution Video Using Gabor Filter \& Adaptive } \\
\text { Histogram Equalization } \\
\text { (Hendy William Sino, Indrabayu, Intan Sari Areni) }\end{array}$ & $\begin{array}{l}\text { Titanium } 2(4), 13.15- \\
13.30\end{array}$ & 417 \\
\hline 1570526500 & $\begin{array}{l}\text { Improved Ranking Based Collaborative Filtering Using SVD and Borda } \\
\text { Algorithm } \\
\text { (Muhammad Iqbal Ardiansyah Teguh Bharata Adji, Noor Akhmad Setiawan) }\end{array}$ & $\begin{array}{l}\text { Ballroom } 1(3), 10.45- \\
11.00\end{array}$ & 422 \\
\hline 1570526502 & $\begin{array}{l}\text { Word Embedding Comparison for Indonesian Language Sentiment Analysis } \\
\text { (Helmi Imaduddin, Widyawan, Silmi Fauziati) }\end{array}$ & $\begin{array}{l}\text { Ballroom } 2(4), 13.15- \\
13.30\end{array}$ & 426 \\
\hline 1570526507 & HERO: Maximizing Student Potential to Mobilize Community Empowerment & Titanium 1 (1), 13.15- & 431 \\
\hline
\end{tabular}




\begin{tabular}{|c|c|c|c|}
\hline & $\begin{array}{l}\text { Activities Around Campus } \\
\text { (Rohmat Tulloh, Ridha Muldina Negara, Yayan Eka Yudha Prasetya, Sendy } \\
\text { Saputra) }\end{array}$ & 13.30 & \\
\hline 1570526512 & $\begin{array}{l}\text { Backpropagation Implementation To Classify Dysgraphia In Children } \\
\text { (Pratama Wisnu Samodro, Sari Widya Sihwi, Winarno) }\end{array}$ & $\begin{array}{l}\text { Titanium } 1(4), 13.45- \\
14.00\end{array}$ & 437 \\
\hline 1570526525 & $\begin{array}{l}\text { The Repercussions of Game Multiplayer Online Battle Arena } \\
\text { (Sasmoko, Senly Halim, Yasinta Indrianti, Yogi Udjaja, Jurike Moniaga, Brilly } \\
\text { Andro Makalew) }\end{array}$ & $\begin{array}{l}\text { Titanium } 1(3), 10.45- \\
11.00\end{array}$ & 443 \\
\hline 1570526529 & $\begin{array}{l}\text { A Review of Sentiment Analysis for Non-English Language } \\
\text { (Fahim Djatmiko, Ridi Ferdiana, Muhammad Faris) }\end{array}$ & $\begin{array}{l}\text { Ballroom } 2(4), 13.45- \\
14.00\end{array}$ & 448 \\
\hline 1570526533 & $\begin{array}{l}\text { The Influence of User Experience Playerunknown's Battlegrounds Game } \\
\text { Toward Adaptive Learning } \\
\text { (Jurike Moniaga, Bonavensius Yosua Aprilianus Tansil, Sasmoko, Yasinta } \\
\text { Indrianti, Noerlina) }\end{array}$ & $\begin{array}{l}\text { Titanium } 1(3), 11.15- \\
11.30\end{array}$ & 452 \\
\hline 1570526538 & $\begin{array}{l}\text { Implementation of Depth-HOG based Human Upper Body Detection On A } \\
\text { Mini PC Using A Low Cost Stereo Camera } \\
\text { (Bima Sena Bayu Dewantara, Fernando Ardilla, Ardiansyah At Thoriqy) }\end{array}$ & $\begin{array}{l}\text { Ballroom } 1 \text { (2), 15.45- } \\
16.00\end{array}$ & 458 \\
\hline 1570526541 & $\begin{array}{l}\text { Thermal Optimization on Incubator using Fuzzy Inference System based loT } \\
\text { (Renny Rakhmawati, Irianto, Farid Dwi Murdianto, Atabik Luthfi, Aviv Yuniar } \\
\text { Rahman) }\end{array}$ & $\begin{array}{l}\text { Ballroom } 2 \text { (3), 10.30- } \\
10.45\end{array}$ & 464 \\
\hline 1570526544 & $\begin{array}{l}\text { A Neural Network based Approach for Predicting Indonesian Teacher } \\
\text { Engagement Index (ITEI) } \\
\text { (Sucianna Ghadati Rabiha, Sasmoko, Emny Harna Yossy, Yasinta Indrianti) }\end{array}$ & $\begin{array}{l}\text { Titanium } 1(2), 16.30- \\
16.45\end{array}$ & 469 \\
\hline 1570526547 & $\begin{array}{l}\text { Incorporating Information Technology Concept to Sustainable Enterprise } \\
\text { (Erda Guslinar Perdana, Husni S. Sastramihardja, Iping Supriana Suwardi) }\end{array}$ & $\begin{array}{l}\text { Ballroom } 1(3), 10.30- \\
10.45\end{array}$ & 475 \\
\hline 1570526549 & $\begin{array}{l}\text { Auto-Encoding Progressive Generative Adversarial Networks for 3D Multi } \\
\text { Object Scenes } \\
\text { (Vedant Singh, Manan Oza, Himanshu Vaghela, Pratik Kanani) }\end{array}$ & $\begin{array}{l}\text { Ballroom } 2(4), 14.45- \\
15.00\end{array}$ & 481 \\
\hline 1570526552 & $\begin{array}{l}\text { Pneumonia Detection with Deep Convolutional Architecture } \\
\text { (Abdullah Faqih Al Mubarok, Ahmad Habbie Thias, Dominique Jeffrey } \\
\text { Alamaro Maximilianus) }\end{array}$ & $\begin{array}{l}\text { Titanium } 3(2), 15.45- \\
16.00\end{array}$ & 486 \\
\hline 1570526555 & $\begin{array}{l}\text { Audio Signal Transmission over Vehicular Channel with Moving Scatterer } \\
\text { (Jans Hendry, Wahyu Pamungkas, Anggun Fitrian Isnawati, Eka Setia } \\
\text { Nugraha) }\end{array}$ & $\begin{array}{l}\text { Titanium } 2(3), 10.30- \\
10.45\end{array}$ & 490 \\
\hline 1570526557 & $\begin{array}{l}\text { Machine Learning for Data Processing in Vessel Telemetry System: Initial } \\
\text { Study } \\
\text { (Herry Susanto, Gunawan Wibisono) }\end{array}$ & $\begin{array}{l}\text { Titanium } 2 \text { (3), 11.15- } \\
11.30\end{array}$ & 496 \\
\hline 1570526563 & $\begin{array}{l}\text { Indonesian Sign Language (BISINDO) Translation System with ORB for } \\
\text { Bilingual Language } \\
\text { (Rahmatullah Arrizal Pranatadesta, Iping Supriana Suwardi) }\end{array}$ & $\begin{array}{l}\text { Titanium } 1(4), 12.30- \\
12.45\end{array}$ & 502 \\
\hline 1570526573 & $\begin{array}{l}\text { An Efficient Resource Allocation Mechanism for Time-Sensitive Data in Dew } \\
\text { Computing } \\
\text { (M Saddam Hossain Khan, Puloma Roy, Fatema Khanam, Farzana Hannan } \\
\text { Hera, Amit Kumar Das) }\end{array}$ & $\begin{array}{l}\text { Ballroom } 1(4), 14.30- \\
14.45\end{array}$ & 506 \\
\hline 1570526714 & $\begin{array}{l}\text { Comparison Road Safety Education with and without loT to Develop } \\
\text { Perceptual Motor Skills in Early Childhood Children Aged 4-5 } \\
\text { (Mario Nugroho Willyarto, Anggraeni S. Reksodipuro, Ulani Yunus, } \\
\text { Suryadiputra Liawatimena) }\end{array}$ & $\begin{array}{l}\text { Ballroom } 1(4), 14.15- \\
14.30\end{array}$ & 511 \\
\hline 1570526750 & $\begin{array}{l}\text { A Simple RBAC And SSO Architecture for ISONER Framework } \\
\text { (I Made Sukarsa, I Ketut Gede Darma Putra, Nyoman Putra Sastra, Lie Jasa) }\end{array}$ & $\begin{array}{l}\text { Titanium } 2 \text { (3), 10.45- } \\
11.00\end{array}$ & 517 \\
\hline
\end{tabular}




\section{International Conference of Artificial Intelligence and Information Technology 2019 List of Papers Per Room}

Titanium 1

\begin{tabular}{|c|c|c|c|}
\hline Session & Time & Paper Code & Title \\
\hline 1 & $13.00-13.15$ & 1570508359 & $\begin{array}{l}\text { Design of Manufacture Professional Training and Assessment Information } \\
\text { System in The Implementation of PBET (Production Based Education and } \\
\text { Training) Learning Activity Model }\end{array}$ \\
\hline 1 & $13.15-13.30$ & 1570526507 & $\begin{array}{l}\text { HERO: Maximizing Student Potential to Mobilize Community } \\
\text { Empowerment Activities Around Campus }\end{array}$ \\
\hline 1 & $13.30-13.45$ & 1570526480 & Predicting Candidates For Fit And Proper Test Using K-Nearest Neighbor \\
\hline 1 & $13.45-14.00$ & 1570526013 & Adapted Flower Pollination Algorithm for Lecturer-Class Assignment \\
\hline 1 & $14.00-14.15$ & 1570518440 & Predictive Analytics for Predicting Customer Behavior \\
\hline 1 & $14.15-14.30$ & 1570519077 & Fluid Simulation Based on Material Point Method with Neural Network \\
\hline 1 & $14.30-14.45$ & 1570518796 & $\begin{array}{l}\text { Decision Support Systems to Determining Programme for Students Using } \\
\text { DBSCAN And Naive Bayes }\end{array}$ \\
\hline 2 & $15.30-15.45$ & 1570515432 & $\begin{array}{l}\text { Big Data Analytics: Estimation of Destination for Users of Bus Rapid Transit } \\
\text { (BRT) Public Transportation in Jakarta }\end{array}$ \\
\hline 2 & $15.45-16.00$ & 1570526222 & $\begin{array}{l}\text { Deep Learning-Based Patient Visits Forecasting Using Long Short Term } \\
\text { Memory }\end{array}$ \\
\hline 2 & $16.00-16.15$ & 1570526304 & $\begin{array}{l}\text { Customizable Dynamic Hand Gesture recognition System for Motor } \\
\text { Impaired people using Siamese neural network }\end{array}$ \\
\hline 2 & $16.15-16.30$ & 1570526319 & $\begin{array}{l}\text { Use of ARIMA Method To Predict The Number of Train Passenger In } \\
\text { Malang City }\end{array}$ \\
\hline 2 & $16.30-16.45$ & 1570526544 & $\begin{array}{l}\text { A Neural Network based Approach for Predicting Indonesian Teacher } \\
\text { Engagement Index (ITEI) }\end{array}$ \\
\hline 2 & $16.45-17.00$ & 1570526452 & $\begin{array}{l}\text { Study on a Train- and Bus-based Delay-Tolerant Networks: Scheduled } \\
\text { Mobility and Impact on Routing }\end{array}$ \\
\hline 3 & $10.30-10.45$ & 1570526412 & The Effect of Game Experience from Counter-Strike: Global Offensive \\
\hline 3 & $10.45-11.00$ & 1570526525 & The Repercussions of Game Multiplayer Online Battle Arena \\
\hline 3 & $11.00-11.15$ & 1570516332 & Gamification of Mobile-based Japanese Language Shadowing \\
\hline 3 & $11.15-11.30$ & 1570526533 & $\begin{array}{l}\text { The Influence of User Experience Playerunknown's Battlegrounds Game } \\
\text { Toward Adaptive Learning }\end{array}$ \\
\hline 4 & $12.30-12.45$ & 1570526563 & $\begin{array}{l}\text { Indonesian Sign Language (BISINDO) Translation System with ORB for } \\
\text { Bilingual Language }\end{array}$ \\
\hline 4 & $12.45-13.00$ & 1570516556 & $\begin{array}{l}\text { A Preliminary Performance Evaluation of Population-Based Algorithms in } \\
\text { VANET }\end{array}$ \\
\hline 4 & $13.00-13.15$ & 1570523658 & Rough-Regression for Categorical Data Prediction based on Case Study \\
\hline 4 & $13.15-13.30$ & 1570524898 & $\begin{array}{l}\text { FHC-Optimization Model for Deciding the Objective Hajj Pilgrims to } \\
\text { Restricted Quota (Case Study: Hajj Pilgrimage Procedure in Indonesia) }\end{array}$ \\
\hline 4 & $13.30-13.45$ & 1570524566 & $\begin{array}{l}\text { Improved Particle Swarm Optimization By Fast Simulated Annealing } \\
\text { Algorithm }\end{array}$ \\
\hline 4 & $13.45-14.00$ & 1570526512 & Backpropagation Implementation To Classify Dysgraphia In Children \\
\hline 4 & $14.00-14.15$ & 1570484576 & $\begin{array}{l}\text { A comparative study on variational autoencoders and generative } \\
\text { adversarial network }\end{array}$ \\
\hline 4 & $14.15-14.30$ & 1570511144 & Design and Development Meeting Schedule Management Application using \\
\hline
\end{tabular}

xxxii 


\begin{tabular}{|l|l|l|l|}
\hline & & & the RAD Method \\
\hline 4 & $14.30-14.45$ & 1570520869 & Designing IT-based Skills and Competency Learning System \\
\hline
\end{tabular}

\section{Titanium 2}

\begin{tabular}{|c|c|c|c|}
\hline Session & Time & Paper Code & Title \\
\hline 1 & $13.00-13.15$ & 1570512443 & $\begin{array}{l}\text { The Priority of Tourism Destinations Development using 6AsTD Framework } \\
\text { and TOPSIS }\end{array}$ \\
\hline 1 & $13.15-13.30$ & 1570512569 & $\begin{array}{l}\text { Improve Smart Waste Management to Preserve Tourist Attractions } \\
\text { Yogyakarta in loT Environment }\end{array}$ \\
\hline 1 & $13.30-13.45$ & 1570514049 & Review of Benefit Using Gamification Element for Countryside Tourism \\
\hline 1 & $13.45-14.00$ & 1570507038 & Social Media Prototyping for Web-based Property Business \\
\hline 1 & $14.00-14.15$ & 1570512457 & Systematic Literature Review of Profiling Analysis Based on Social Media \\
\hline 1 & $14.15-14.30$ & 1570526214 & $\begin{array}{l}\text { Harmonics Reduction for Four-Leg Distribution Network-Connected Single } \\
\text { Phase Transformerless PV Inverter System Using Diagonal Recurrent Neural } \\
\text { Network }\end{array}$ \\
\hline 1 & $14.30-14.45$ & 1570513290 & $\begin{array}{l}\text { Automated Test Suite for Regression Testing Based On Serenity } \\
\text { Framework: A Case Study }\end{array}$ \\
\hline 2 & $15.30-15.45$ & 1570513614 & Complexity Reduction for Multiview HEVC Codec Using FPGA \\
\hline 2 & $15.45-16.00$ & 1570497900 & Semi-Supervised Image-to-Image Translation \\
\hline 2 & $16.00-16.15$ & 1570497821 & $\begin{array}{l}\text { Face Detection using Haar Cascades to Filter Selfie Face Image on } \\
\text { Instagram }\end{array}$ \\
\hline 2 & $16.15-16.30$ & 1570512658 & A classification approach of emotional reactions while driving a vehicle \\
\hline 2 & $16.30-16.45$ & 1570523975 & $\begin{array}{l}\text { Image Based Leaf Area Measurement Method Using Artificial Neural } \\
\text { Network }\end{array}$ \\
\hline 2 & $16.45-17.00$ & 1570525893 & $\begin{array}{l}\text { Detection of Anomalies in Citrus Leaves Using Digital Image Processing and } \\
\mathrm{T}^{2} \text { Hotelling Multivariate Control Chart }\end{array}$ \\
\hline 3 & $10.30-10.45$ & 1570526555 & Audio Signal Transmission over Vehicular Channel with Moving Scatterer \\
\hline 3 & $10.45-11.00$ & 1570526750 & A Simple RBAC And SSO Architecture for ISONER Framework \\
\hline 3 & $11.00-11.15$ & 1570508178 & Simple Implementation of Fuzzy Controller for Low Cost Microcontroller \\
\hline 3 & $11.15-11.30$ & 1570526557 & $\begin{array}{l}\text { Machine Learning for Data Processing in Vessel Telemetry System: Initial } \\
\text { Study }\end{array}$ \\
\hline 4 & $12.30-12.45$ & 1570512995 & Audio Steganography Using Lifting Wavelet Transform and Dynamic Key \\
\hline 4 & $12.45-13.00$ & 1570510797 & $\begin{array}{l}\text { Visual Servoing using Mixed Sensitivity Ho Control for Yaw-Pitch Camera } \\
\text { Platform }\end{array}$ \\
\hline 4 & $13.00-13.15$ & 1570510791 & $\begin{array}{l}\text { Real-time Moving Object Video Tracking using Support Vector Machines } \\
\text { for Visual Servo Application }\end{array}$ \\
\hline 4 & $13.15-13.30$ & 1570526499 & $\begin{array}{l}\text { Face Recognition of Low-Resolution Video Using Gabor Filter \& Adaptive } \\
\text { Histogram Equalization }\end{array}$ \\
\hline 4 & $13.30-13.45$ & 1570526192 & $\begin{array}{l}\text { Digital Overcurrent Relay Implementation With Non-Standard Inverse } \\
\text { Curve Modelling Using Adaptive Neuro Fuzzy Inference System }\end{array}$ \\
\hline 4 & $13.45-14.00$ & 1570526423 & $\begin{array}{l}\text { Dynamic Background Video Forgery Detection using Gaussian Mixture } \\
\text { Model }\end{array}$ \\
\hline 4 & $14.00-14.15$ & 1570523474 & $\begin{array}{l}\text { Comparison Of Distance Methods In K-Means Algorithm For Determining } \\
\text { Village Status In Bekasi District }\end{array}$ \\
\hline 4 & $14.15-14.30$ & 1570526180 & Fuzzy Coordinator based Al for Dynamic Difficulty Adjustment in Starcraft 2 \\
\hline
\end{tabular}




\begin{tabular}{|c|c|c|l|}
\hline 4 & $14.30-14.45$ & 1570514074 & $\begin{array}{l}\text { The Classification of the Movie Genre's based on Synopsis of the } \\
\text { Indonesian Film }\end{array}$ \\
\hline
\end{tabular}

Titanium 3

\begin{tabular}{|c|c|c|c|}
\hline Session & Time & Paper Code & Title \\
\hline 1 & $13.00-13.15$ & 1570512696 & IoT-Based Smart and Healthy Wardrobe System \\
\hline 1 & $13.15-13.30$ & 1570512578 & $\begin{array}{l}\text { Smart Kost: Ubiquitous Boarding House Controlling and Monitoring System } \\
\text { in Industry } 4.0\end{array}$ \\
\hline 1 & $13.30-13.45$ & 1570512583 & $\begin{array}{l}\text { IoT Based: Improving Control System For High-Quality Beef in } \\
\text { Supermarkets }\end{array}$ \\
\hline 1 & $13.45-14.00$ & 1570512614 & IOT: Improved Home Energy Control System Based on Consumer Behavior \\
\hline 1 & $14.00-14.15$ & 1570512722 & IoT Based: Hydroponic Using Drip Non-Circulation System for Paprika \\
\hline 1 & $14.15-14.30$ & 1570512735 & $\begin{array}{l}\text { Smart hydroponic farming with loT-based climate and nutrient } \\
\text { manipulation system }\end{array}$ \\
\hline 1 & $14.30-14.45$ & 1570518672 & $\begin{array}{l}\text { Internet of Things: Roboboat for Water Area Monitoring using } 4 \text { G network } \\
\text { and Google Firebase }\end{array}$ \\
\hline 2 & $15.30-15.45$ & 1570526455 & $\begin{array}{l}\text { Automated Segmentation of Breast Tissue and Pectoral Muscle in Digital } \\
\text { Mammography }\end{array}$ \\
\hline 2 & $15.45-16.00$ & 1570526552 & Pneumonia Detection with Deep Convolutional Architecture \\
\hline 2 & $16.00-16.15$ & 1570516699 & $\begin{array}{l}\text { Classification of Premature Ventricular Contraction based on ECG Signal } \\
\text { using Multiorder Rényi Entropy }\end{array}$ \\
\hline 2 & $16.15-16.30$ & 1570513732 & $\begin{array}{l}\text { EEG-based Mental Fatigue Detection Using Cognitive Tests and RVM } \\
\text { Classification }\end{array}$ \\
\hline 2 & $16.30-16.45$ & 1570509093 & Extraction of Skull and Face Surfaces from CT Images \\
\hline 2 & $16.45-17.00$ & 1570526401 & $\begin{array}{l}\text { Heart Rate Estimation from Wrist-Type Photoplethysmographic Signals } \\
\text { Corrupted by Intense Motion Artifacts using NLMS Adaptive Filter and } \\
\text { Spectral Peak Tracking }\end{array}$ \\
\hline
\end{tabular}

\section{Ballroom 1}

\begin{tabular}{|c|c|c|c|}
\hline Session & Time & Paper Code & Title \\
\hline 2 & $15.30-15.45$ & 1570521650 & $\begin{array}{l}\text { Determining The Threshold Value for Identification of The Goblet Cells in } \\
\text { Chicken Small Intestine }\end{array}$ \\
\hline 2 & $15.45-16.00$ & 1570526538 & $\begin{array}{l}\text { Implementation of Depth-HOG based Human Upper Body Detection On A } \\
\text { Mini PC Using A Low Cost Stereo Camera }\end{array}$ \\
\hline 2 & $16.00-16.15$ & 1570526466 & $\begin{array}{l}\text { Determining Banana Types and Ripeness from Image using Machine } \\
\text { Learning Methods }\end{array}$ \\
\hline 2 & $16.15-16.30$ & 1570526453 & $\begin{array}{l}\text { A Study on Part Affinity Fields Implementation for Human Pose Estimation } \\
\text { with Deep Neural Network }\end{array}$ \\
\hline 2 & $16.30-16.45$ & 1570524081 & $\begin{array}{l}\text { Contrast-enhanced Based on Abdominal Kernels for CT Image Noise } \\
\text { Reduction }\end{array}$ \\
\hline 2 & $16.45-17.00$ & 1570513593 & Clutter Mitigation Technique on OFDM MIMO Radar \\
\hline 3 & $10.30-10.45$ & 1570526547 & Incorporating Information Technology Concept to Sustainable Enterprise \\
\hline 3 & $10.45-11.00$ & 1570526500 & $\begin{array}{l}\text { Improved Ranking Based Collaborative Filtering Using SVD and Borda } \\
\text { Algorithm }\end{array}$ \\
\hline 3 & $11.00-11.15$ & 1570511011 & $\begin{array}{l}\text { Automatic Lecture Video Content Summarization with Attention-based } \\
\text { Recurrent Neural Network }\end{array}$ \\
\hline 3 & $11.15-11.30$ & 1570522671 & $\begin{array}{l}\text { Traffic Sign Image Recognition Using Gabor Wavelet and Principle } \\
\text { Component Analysis }\end{array}$ \\
\hline 4 & $12.30-12.45$ & 1570512694 & $\begin{array}{l}\text { Determining the Neural Network Topology from the Viewpoint of Kuhn's } \\
\text { Philosophy and Popper's Philosophy }\end{array}$ \\
\hline
\end{tabular}




\begin{tabular}{|c|c|c|l|}
\hline 4 & $12.45-13.00$ & 1570497875 & MREAK: Morphological Retina Keypoint Descriptor \\
\hline 4 & $13.00-13.15$ & 1570512201 & $\begin{array}{l}\text { The Influence of Sampling Frequency on Guitar Chord Recognition using } \\
\text { DST Based Segment Averaging }\end{array}$ \\
\hline 4 & $13.15-13.30$ & 1570513608 & $\begin{array}{l}\text { Forming Formation of Particle Swarm using Artificial Neural Network Self } \\
\text { Organizing Map (ANN-SOM) with 2-leveled Strategy }\end{array}$ \\
\hline 4 & $13.30-13.45$ & 1570513712 & $\begin{array}{l}\text { Modified Ant Colony Algorithm For Swarm Multi Agent Exploration on } \\
\text { Target Searching in Unknown Environment }\end{array}$ \\
\hline 4 & $13.45-14.00$ & 1570513896 & Parameter Identifiability of Phased-MIMO Radar \\
\hline 4 & $14.00-14.15$ & 1570513597 & $\begin{array}{l}\text { Switching Formation and Topology in Cooperative Multi-Agent Source } \\
\text { Seeking Using Gradient Estimation }\end{array}$ \\
\hline 4 & $14.15-14.30$ & 1570526714 & $\begin{array}{l}\text { Comparison Road Safety Education with and without loT to Develop } \\
\text { Perceptual Motor Skills in Early Childhood Children Aged 4-5 }\end{array}$ \\
\hline & $14.30-14.45$ & 1570526573 & $\begin{array}{l}\text { An Efficient Resource Allocation Mechanism for Time-Sensitive Data in Dew } \\
\text { Computing }\end{array}$ \\
\hline
\end{tabular}

\section{Ballroom 2}

\begin{tabular}{|c|c|c|c|}
\hline Session & Time & Paper Code & Title \\
\hline 3 & $10.30-10.45$ & 1570526541 & Thermal Optimization on Incubator using Fuzzy Inference System based IoT \\
\hline 3 & $10.45-11.00$ & 1570512562 & NDNization of IP Network Based On Communication Flow Model \\
\hline 3 & $11.00-11.15$ & 1570513741 & $\begin{array}{l}\text { Reinforcement Point and Fuzzy Input Design of Fuzzy Q-Learning for } \\
\text { Mobile Robot Navigation System }\end{array}$ \\
\hline 3 & $11.15-11.30$ & 1570513710 & Trajectory Tracking Automated Guided Vehicle using Fuzzy Controller \\
\hline 4 & $12.30-12.45$ & 1570515288 & A Study of Text Classification for Indonesian News Article \\
\hline 4 & $12.45-13.00$ & 1570523931 & $\begin{array}{l}\text { A Comparison of the Use of Several Different Resources on Lexicon Based } \\
\text { Indonesian Sentiment Analysis on App Review Dataset }\end{array}$ \\
\hline 4 & $13.00-13.15$ & 1570526385 & $\begin{array}{l}\text { Sentiment Analysis In Twitter Using Lexicon Based and Polarity } \\
\text { Multiplication }\end{array}$ \\
\hline 4 & $13.15-13.30$ & 1570526502 & Word Embedding Comparison for Indonesian Language Sentiment Analysis \\
\hline 4 & $13.30-13.45$ & 1570526281 & Offensive Language Detection using Artificial Neural Network \\
\hline 4 & $13.45-14.00$ & 1570526529 & A Review of Sentiment Analysis for Non-English Language \\
\hline 4 & $14.00-14.15$ & 1570521672 & $\begin{array}{l}\text { Information Retrieval System for Searching JSON Files with Vector Space } \\
\text { Model Method }\end{array}$ \\
\hline 4 & $14.15-14.30$ & 1570526218 & Melanoma Classification Using Texture and Wavelet Analysis \\
\hline 4 & $14.30-14.45$ & 1570526456 & $\begin{array}{l}\text { Proportional-Derivative Control for Quadrotor Stabilization under Inertia } \\
\text { Perturbation }\end{array}$ \\
\hline 4 & $14.45-15.00$ & 1570526549 & $\begin{array}{l}\text { Auto-Encoding Progressive Generative Adversarial Networks For 3D Multi } \\
\text { Object Scenes }\end{array}$ \\
\hline
\end{tabular}




\section{Proceeding - 2019 International Conference of Artificial Intelligence and Information Technology, ICAIIT 2019}

\begin{tabular}{|c|c|c|c|}
\hline COUNTRY & SUBJECT AREA AND CATEGORY & PUBLISHER & H-INDEX \\
\hline $\begin{array}{l}\text { United States } \\
\qquad \begin{array}{l}\text { Universities and research } \\
\text { institutions in United States }\end{array}\end{array}$ & $\begin{array}{l}\text { Computer Science } \\
\text { Artificial Intelligence } \\
\text { Computer Networks and } \\
\text { Communications } \\
\text { Computer Science } \\
\text { Applications } \\
\text { Information Systems } \\
\text { Signal Processing } \\
\text { Mathematics } \\
\text { Control and Optimization }\end{array}$ & & 4 \\
\hline PUBLICATION TYPE & ISSN & COVERAGE & \\
\hline Conferences and Proceedings & - & 2019 & \\
\hline
\end{tabular}



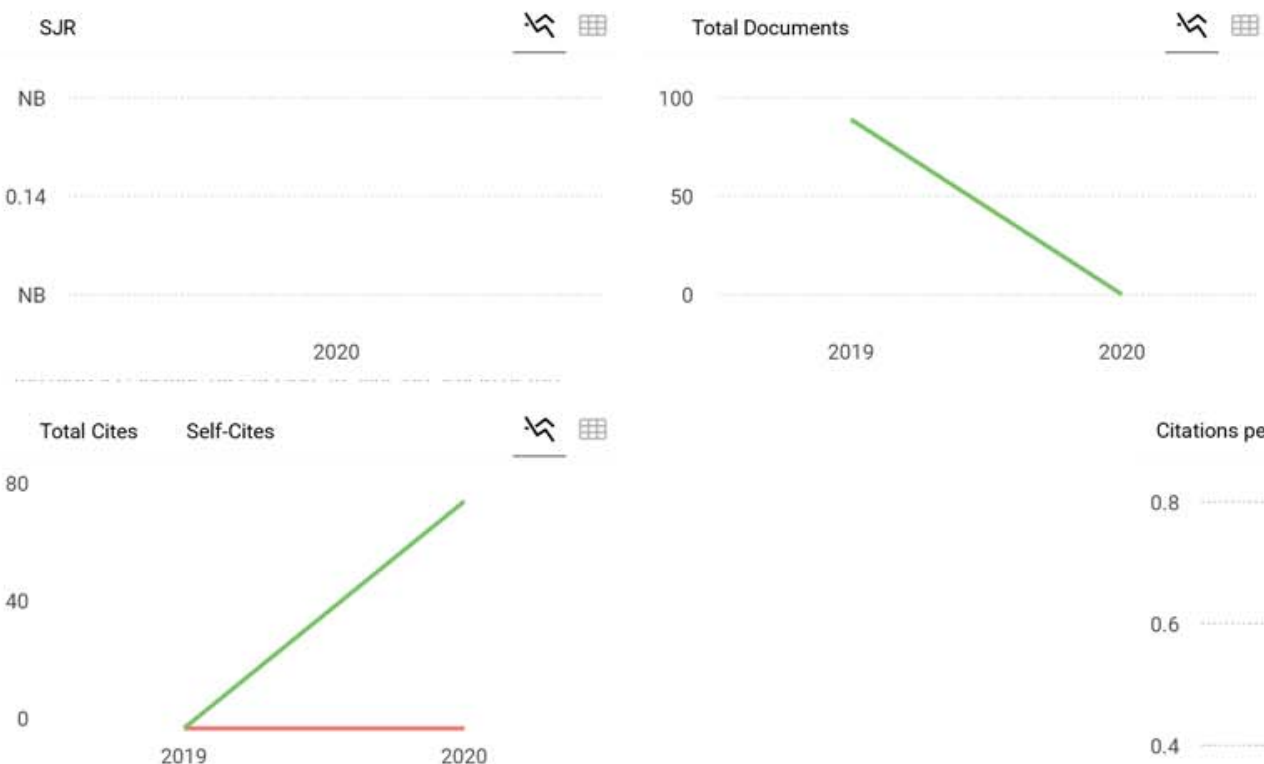

External Cites per Doc Cites per Doc औ囲

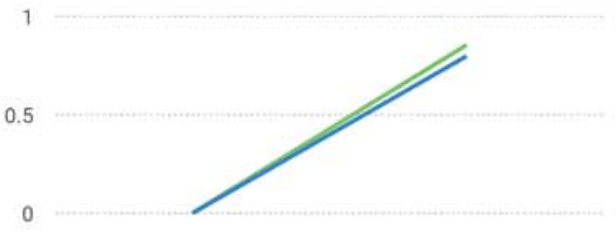

2019

2020
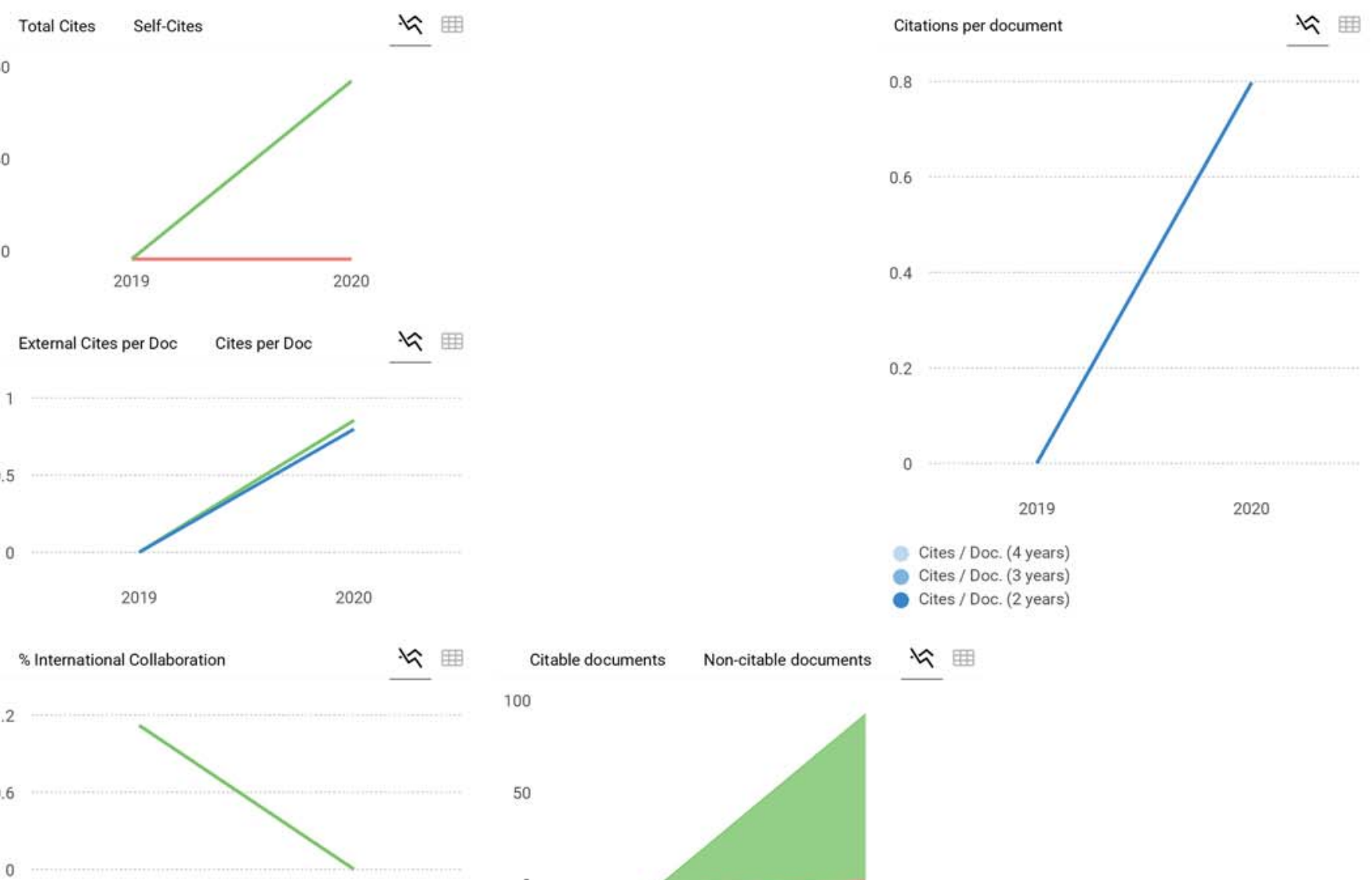

2019

2020

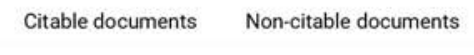

ネ柬

100

50

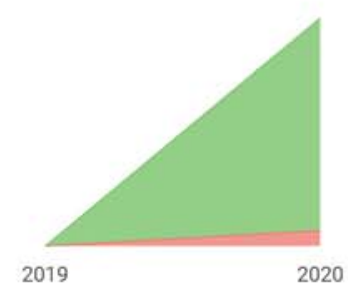

Cited documents Uncited documents 細

100

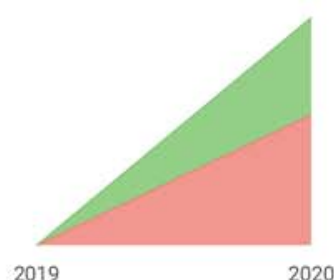

Proceeding - 2019

$\leftarrow$ Show this widget in

International Conference of.. your own website

Not yet assigned

quartile

SIR 2020

0.14

Just copy the code below

and paste within your htm

code:

powered by scimagojir.com

$<$ href="https://www.scim:

2020

\section{G SCImago Graphica}

Explore, visually

communicate and make sense of data with our new free tool.

Get it

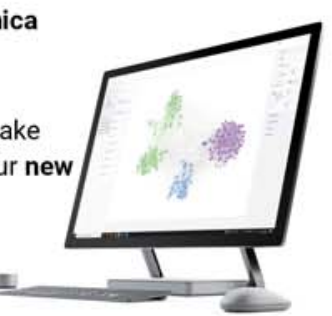


Metrics based on Scopus ${ }^{\circledR}$ data as of April 2021

\author{
Leave a comment \\ Name \\ Email \\ (will not be published)
}

The users of Scimago Journal \& Country Rank have the possibility to dialogue through comments linked to a specific journal. The purpose is to have a forum in which general doubts about the processes of publication in the journal, experiences and other issues derived from the publication of papers are resolved. For topics on particular articles, maintain the dialogue through the usual channels with your editor. 
Developed by:

Powered by:

SCImago

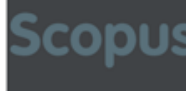

Follow us on @ScimagoJR

Scimago Lab, Copyright 2007-2020. Data Source: Scopus ${ }^{\circledR}$ 


\section{Document details - Image based leaf area measurement method using artificial neural network}

\author{
1 of 1 \\ $\rightarrow$ Export $\Perp$ Download More...>
}

Proceeding - 2019 International Conference of Artificial Intelligence and Information Technology, ICAIIT 2019

March 2019, Article number 8834500, Pages 288-292

lst International Conference of Artificial Intelligence and Information Technology, ICAIIT 2019; Platinum Adisucipto Hotel and Conference CenterYogyakarta; Indonesia; 13 March 2019 through 15 March 2019; Category numberCFP19JVE-ART; Code 151944

Image based leaf area measurement method using artificial neural network(Conference Paper)(Open Access)

Siswantoro, J., Artadana, I.B.M.

${ }^{a}$ Department of Informatics Engineering Universitas Surabaya, Surabaya, Indonesia

${ }^{b}$ Department of Biology Universitas Surabaya, Surabaya, Indonesia

Abstract

Leaf area is an important parameter in plant monitoring. An automatic method for measuring leaf area is needed to obtain accurate result. In this paper, a method for automatic leaf area measurement from image is proposed. The method captured the image of leaf without object reference. Four features were extracted from the image and used as input to artificial neural network for estimating leaf area. The experiment results show that the proposed method can measure leaf area with mean absolute relative error less than $1 \%$ and less computational time. (C) 2019 IEEE.

SciVal Topic Prominence (i)

Topic: Leaf Area | Sum of Squares | Coefficient of Determination

Prominence percentile: $77.205 \quad$ (i)

Author keywords

Artificial neural network Automatic Image processing leaf area measurement

Indexed keywords

Engineering Geodesy Neural networks Surveying

controlled terms:

Engineering uncontrolled terms

Automatic Automatic method Computational time Object reference Plant monitoring Relative errors

Engineering main heading:

Image processing

Funding details

Funding sponsor

Funding number

Acronym
Cited by 2 documents

Sabouri, H. , Sajadi, S.J., Jafarzadeh, M.R.

Image processing and prediction of leaf area in cereals: A comparison of artificial neural networks, an adaptive neurofuzzy inference system, and regression methods

(2021) Crop Science

Tu, L.-F. , Peng, Q. , Li, C.-S.

2D in Situ Method for Measuring Plant Leaf Area with Camera Correction and Background Color Calibration

(2021) Scientific Programming

View details of all 2 citations

Inform me when this document is cited in Scopus:

$\begin{array}{ll}\text { Set citation } & \text { Set citation } \\ \text { alert }> & \text { feed }>\end{array}$

Related documents

Find more related documents in Scopus based on:

Authors > Keywords >
Universitas Surabaya

033/Lit/LPPM-01/FT/V/2018 
ISBN: 978-153868448-1

Source Type: Conference Proceeding

Original language: English
DOI: 10.1109/ICAIIT.2019.8834500

Document Type: Conference Paper

Publisher: Institute of Electrical and Electronics Engineers Inc.

(C) Copyright 2020 Elsevier B.V., All rights reserved. 


\section{About Scopus}

\section{What is Scopus}

Content coverage

Scopus blog

Scopus API

Privacy matters

\section{Language}

日本語に切り替える

切换到简体中文

切換到繁體中文

Русский язык

\section{Customer Service}

Help

Contact us

\section{ELSEVIER Terms and conditions $\pi$ Privacy policy $\pi$}

Copyright (C Elsevier B.V त. All rights reserved. Scopus ${ }^{\circledR}$ is a registered trademark of Elsevier B.V.

We use cookies to help provide and enhance our service and tailor content. By continuing, you agree to the use

QRELX

of cookies. 